ARTICLE

DOI: $10.1038 / \mathrm{s} 41467-018-03072-8$

\title{
Ultraconserved element uc.372 drives hepatic lipid accumulation by suppressing miR-195/miR4668 maturation
}

Jun Guo', Weiwei Fang ${ }^{1,2}$, Libo Sun ${ }^{3}$, Yonggang Lu¹, Lin Dou¹, Xiuqing Huang ${ }^{1}$, Weiqing Tang ${ }^{1,4}$, Liqing $\mathrm{Yu}^{4} \&$ Jian $\mathrm{Li}^{1,2}$

Ultraconserved (uc) RNAs, a class of long non-coding RNAs (IncRNAs), are conserved across humans, mice, and rats, but the physiological significance and pathological role of ucRNAs is largely unknown. Here we show that uc.372 is upregulated in the livers of $\mathrm{db} / \mathrm{db}$ mice, HFDfed mice, and NAFLD patients. Gain-of-function and loss-of-function studies indicate that uc.372 drives hepatic lipid accumulation in mice by promoting lipogenesis. We further demonstrate that uc.372 binds to pri-miR-195/pri-miR-4668 and suppresses maturation of miR-195/miR-4668 to regulate expression of genes related to lipid synthesis and uptake, including ACC, FAS, SCD1, and CD36. Finally, we identify that uc.372 is located downstream of the insulinoma-associated 2 (INSM2) gene that is transcriptionally activated by upstream transcription factor 1 (USF1). Our findings reveal a novel mechanism by which uc.372 drives hepatic steatosis through inhibition of miR-195/miR-4668 maturation to relieve miR-195/ miR-4668-mediated suppression of functional target gene expression.

\footnotetext{
${ }^{1}$ The MOH Key Laboratory of Geriatrics, Beijing Hospital, National Center of Gerontology, Beijing 100730, China. ${ }^{2}$ Graduate School of Peking Union Medical College and Chinese Academy of Medical Sciences, Beijing 100730, China. ${ }^{3}$ Department of Hepatobiliay Surgery and You-An Liver Transplantation Center, Beijing You-An Hospital, Capital Medical University, Beijing 100069, China. ${ }^{4}$ Center for Molecular and Translational Medicine, Institute for Biomedical Sciences, Georgia State University, Atlanta, GA 30303, USA. Jun Guo and Weiwei Fang authors contributed equally to this work. Correspondence and requests for materials should be addressed to L.Y. (email: LYU68@gsu.edu) or to J.L. (email: lijian@bjhmoh.cn)
} 
A s the most prevalent chronic liver disease, the pathogenesis of non-alcoholic fatty liver disease (NAFLD) is complicated and multi-faceted. According to the doublehit theory, the first hit of NAFLD is the accumulation of abnormal triglycerides in hepatocytes, thereby resulting in the second hit in which inflammatory mediators further lead to liver injury, inflammation, and fibrosis ${ }^{1,2}$. To improve our understanding of NAFLD pathogenesis, ongoing efforts has been mainly focused on protein-coding genes ${ }^{3-5}$. However, the majority of the human genome produces functional non-coding $\mathrm{RNA}^{6}$. Increasing evidence suggests important biological, developmental, and pathological roles for non-coding RNA through mechanisms, including cis regulation at enhancers, chromatin reprogramming, and mRNA processing at the posttranscriptional level ${ }^{7,8}$.

Although long non-coding RNAs (lncRNAs) were previously considered to be transcriptional "noise," accumulating evidence has identified a biological function for $\operatorname{lncRNAs}{ }^{9,10}$. Recent studies raise the possibility that the appearance of $\ln \mathrm{R} N A s$ plays an important regulatory role in the pathogenesis of human diseases ${ }^{11,12}$. However, the functional role of $\operatorname{lncRNAs}$ remains largely unknown ${ }^{13}$.

LncRNAs, particularly highly conserved ones, are postulated to undergo active regulation and may have a cellular function ${ }^{14,15}$. The ultraconservation of some lncRNAs came to light after a genome-wide survey identified 481 lncRNAs longer than $200 \mathrm{bp}$ in length with $100 \%$ identity across the human, mouse, and rat genomes ${ }^{16}$. These highly conserved lncRNAs have been termed ultraconserved elements (UCEs) ${ }^{16}$. Functional analysis indicates that UCEs are distributed in clusters in regions that are predicted to code for transcription factors and developmental genes ${ }^{17,18}$. These ultraconserved regions have been suggested to play critical roles in DNA binding, RNA processing, and transcription ${ }^{17,19}$. However, our understanding of these elements remains limited. Recently, it has been shown that the expression pattern of transcribed ultraconserved RNAs (ucRNAs) is altered in many human tumors, indicating their potential involvement in cancer progression $^{20-22}$. For example, aberrant expression of UCE 73 (uc.73) regulates apoptosis and cellular proliferation in colorectal cancer cells ${ }^{20}$. Several ucRNAs are tightly correlated with clinical prognostic factors in neuroblastoma ${ }^{23}$. Despite these emerging findings, we still lack knowledge about the functional role of ucRNAs in NAFLD progression. In this study, we suggested a novel mechanism by which uc.372 drives hepatic steatosis through inhibition of miR-195/miR-4668 maturation to relieve miR-195/miR-4668-mediated suppression of functional target gene related to lipid synthesis, including acetyl-CoA carboxylase (ACC), fatty acid synthase (FAS), stearoyl-CoA desaturase 1 (SCD1), and genes related to lipid uptake such as CD36, leading to hepatic lipid accumulation. We propose that uc.372 inhibitors may represent potential therapeutic agents for NAFLD.

In sum, therefore, we have identified an ucRNA-based miRNA maturation regulatory pathway that promotes lipid accumulation in liver cells, highlighting the role that ucRNAs play in NAFLD progression.

\section{Results}

Upregulation of uc.372 in the livers of $\mathrm{db} / \mathrm{db}$ and high-fat dietfed mice. To identify ucRNAs that are potentially involved in hepatic lipid metabolism, we first searched for ucRNAs that are upregulated in the liver of $\mathrm{db} / \mathrm{db}$ mice. LncRNA-wide expression profiling identified 16 ucRNAs, representing 3\% of all ucRNAs analyzed, that were aberrantly increased by $\sim 6.1$ - to $\sim 30.4$-fold in the liver of $\mathrm{db} / \mathrm{db}$ mice accompanied by abnormal hepatic lipid accumulation, compared to the livers of wild-type (WT) mice
(Fig. 1a, Supplementary Fig. 1a, b). We further assessed changes in these identified ucRNAs by real-time PCR analysis. As shown in Fig. 1b, among the identified 16 ucRNAs, 5 ucRNAs (uc.348, uc.372, uc.94, uc.157, and uc.436) were markedly increased in the liver of $\mathrm{db} / \mathrm{db}$ mice $(n=5)$. Moreover, we determined levels of 5 ucRNAs in the liver of high-fat diet (HFD)-fed mice and found that the level of uc.372, but not uc.348, uc.94, uc.157, and uc.436, was increased by $4.4 \pm 0.4$-fold in the liver of these mice accompanied by abnormal hepatic lipid accumulation (Fig. 1c and Supplementary Fig. 1c, d). Next, by analysis of uc.372 distribution in various tissues, we found that uc.372 was widely expressed in mouse organs (Fig. 1d). We hypothesized that if lncRNA controls lipid metabolism, its expression pattern might be affected by changes in metabolic state. In mice subjected to a HFD, uc.372 expression was abundantly increased in metabolismrelated organs, including muscle, liver, heart, and fat (Fig. 1e), suggesting that uc.372 is modulated by the metabolic milieu in vivo, thereby acting as a potential metabolic regulator in mice.

To determine whether uc.372 is regulated by the metabolic milieu in vitro, human HepG2 hepatocytes were stimulated with $33.3 \mathrm{mmol} / \mathrm{L}$ glucose for $48 \mathrm{~h}, 10 \mathrm{nmol} / \mathrm{L}$ tumor necrosis factor (TNF)- $\alpha$ for $24 \mathrm{~h}, 10 \mathrm{nmol} / \mathrm{L}$ interleukin (IL)-6, or $0.25 \mathrm{mmol} / \mathrm{L}$ palmitate for $24 \mathrm{~h}$. As shown in Fig. 1f, palmitate, but not glucose, IL- 6 or TNF- $\alpha$, upregulated uc.372 expression in HepG 2 cells. Collectively, these results imply that uc.372 may be involved in hepatic lipid metabolism.

uc.372 drives hepatic lipid accumulation in mice. Next, we hypothesized that hepatic uc.372 upregulation might contribute to aberrant lipid accumulation. To test this idea, uc.372 was overexpressed in the liver of $\mathrm{C} 57 \mathrm{BL} / 6 \mathrm{~J}$ mice using recombinant adenoviral vectors (Ad-uc.372 m). Seven days after administration of Ad-uc. $372 \mathrm{~m}$ to mice by tail vein injection, uc. 372 was overexpressed in the liver of $\mathrm{C} 57 \mathrm{BL} / 6 \mathrm{~J}$ mice, as indicated by realtime PCR (Fig. 2a). Of note, significantly elevated hepatic triglyceride levels, liver weight, ratio of liver weight to body weight, serum cholesterol, and triglyceride levels (Fig. 2b, c, Supplementary Fig. 2a-c) occurred in Ad-uc.372m-treated C57BL/6J mice. We further investigated whether increased expression of uc.372 in hepatocytes could enhance lipid accumulation. As expected, adenovirus-mediated overexpression of uc.372 in HepG2 cells (Fig. 2d) led to lipid accumulation, as shown by Oil Red $\mathrm{O}$ staining and triglyceride content quantification in hepatocytes (Fig. 2e, f). Moreover, we examined the expression of uc.372 in different tissues after tail vein injection with Aduc. $372 \mathrm{~m}$ by northern blot. Ad-uc.372m injection led to high expression of uc.372 in the liver, but not in other tissues (Supplementary Fig. 2g). These data suggest that uc.372 overexpression causes severe hepatic lipid accumulation in C57BL/6J mice.

To further assess the role of uc.372 in lipid accumulation, we suppressed uc.372 expression in the liver of HFD-fed mice by recombinant adenoviral vectors expressing an uc.372 inhibitor (Ad-uc.372i) (Fig. 2g). Importantly, we found significantly reduced hepatic triglyceride levels, liver weight, ratio of liver weight to body weight, serum cholesterol, and triglyceride levels in HFD-fed mice injected with Ad-uc.372i (Fig. 2h, i, Supplementary Fig. 2d-f). We next inhibited uc.372 expression by transfection of Ad-uc.372i into HepG2 cells pre-treated with a mixture of oleic acid and palmitic acid (O/P mixture, 2:1, M/M) for $24 \mathrm{~h}$ (Fig. 2j). As indicated in Fig. 2k, l, decreased uc.372 expression in hepatocytes attenuated the lipid accumulation induced by the $\mathrm{O} / \mathrm{P}$ mixture. These results reveal that inhibition of uc. 372 has profound lipid-lowering effects in the hepatocytes of HFD-fed mice. 


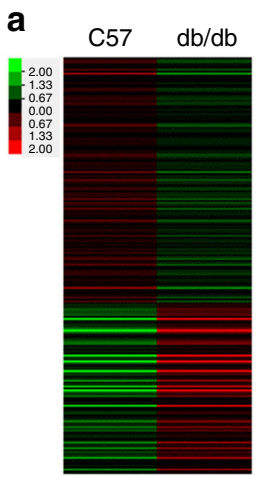

C

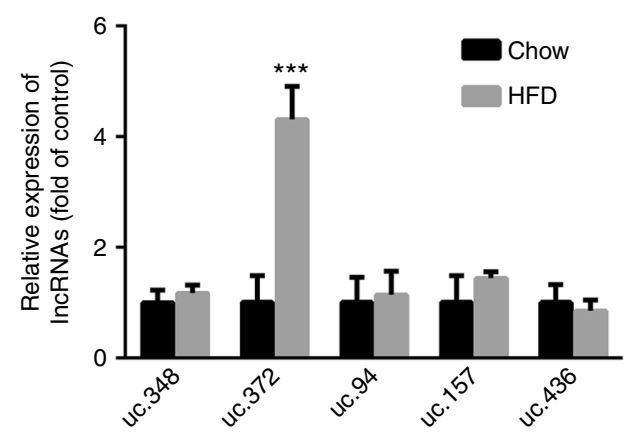

e

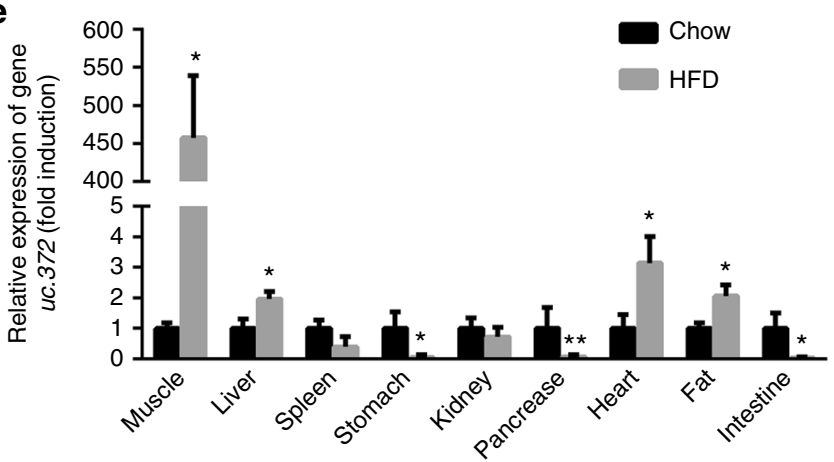

d
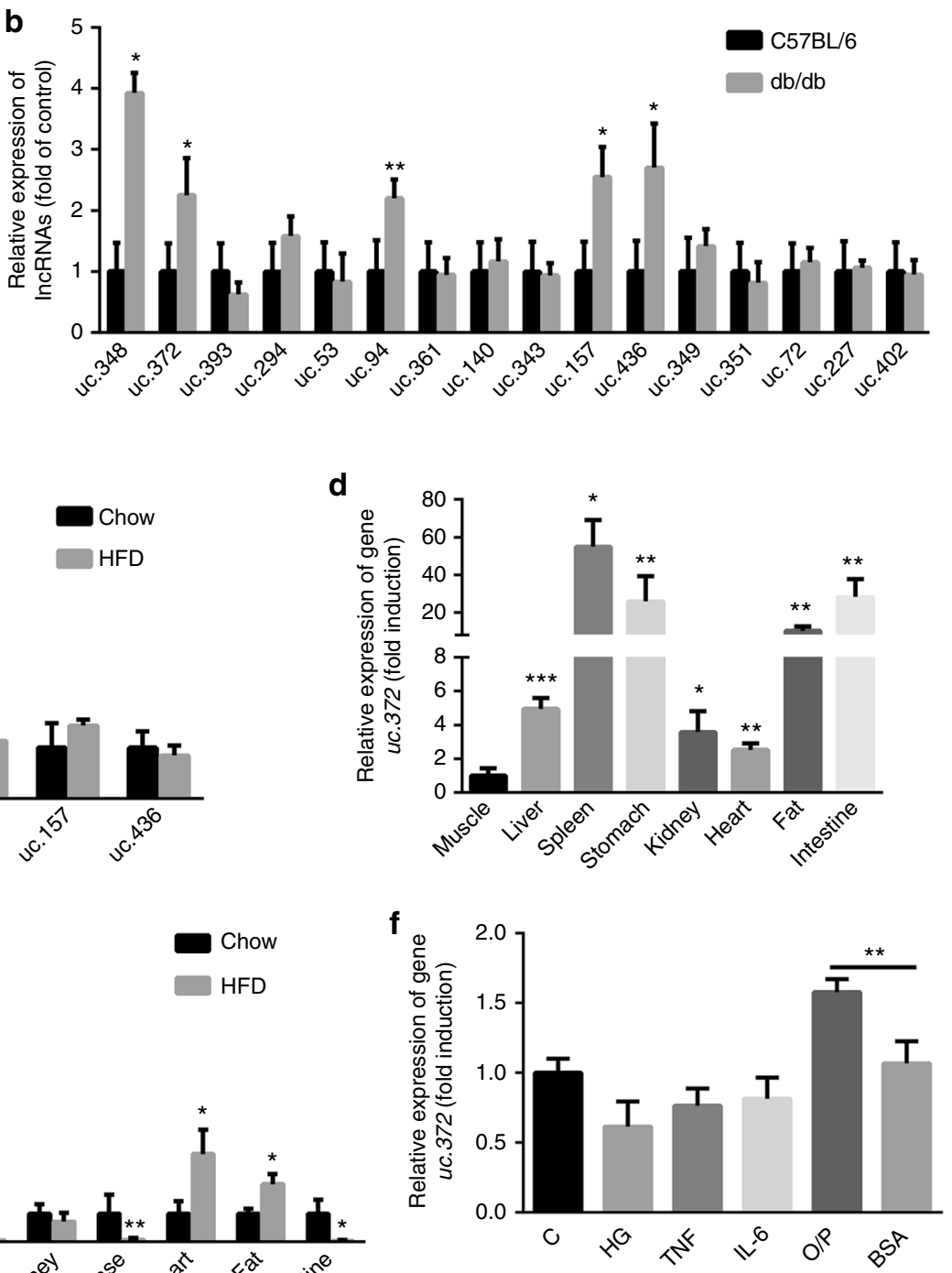

Fig. 1 Identification of uc.372 upregulated in the livers of db/db mice and HFD-fed mice. a LncRNA-wide expression profiling in the liver of 8-week-old male $\mathrm{db} / \mathrm{db}$ mice $(n=5)$. b Expression levels of ucRNAs in the liver of 8-week-old male $\mathrm{db} / \mathrm{db}$ mice analyzed by real-time PCR $(n=5)$. c Expression levels of ucRNAs in the liver of 10-week-HFD-fed mice analyzed by real-time PCR $(n=5)$. d Distribution of uc.372 in various tissues of 6- to 8-week-old C57BL/6J mice $(n=3)$. e Distribution of uc.372 in various tissues of 10-week-HFD-fed mice $(n=5)$. f Expression level of uc. 372 in HepG 2 cells treated with 33.3 mM high glucose (HG), $20 \mathrm{nM}$ tumor necrosis factor (TNF), $20 \mathrm{nM} \mathrm{IL}-6$, or $300 \mu \mathrm{M}$ O/P mixture $(n=3)$. Data are mean $\pm \mathrm{SEM}$; ${ }^{\star} P<0.05$; ${ }^{\star \star} P<0.01$; ${ }^{\star \star \star} P<$ 0.001 vs. control group. (b, c, e Student's t-test; $\mathbf{d}, \mathbf{f}$ analysis of variance (ANOVA))

uc.372 promotes lipid synthesis and uptake in hepatocytes. To explore the underlying mechanism by which uc.372 regulates hepatic lipid metabolism, we assessed the expression levels of genes related to lipid synthesis (ACC, FAS, SCD, SREBP1, and LXR), uptake (Fatp1, Fatp2, Fatp5, Fabp1, and CD36), oxidation (Cpt1a, Scad, Acoxl, and PPAR $\alpha$ ) and secretion ( $a p o B$ and $M t p)$ in HepG2 cells transfected with Ad-uc.372m. We found that expression of genes related to lipid synthesis, including ACC, FAS, and $S C D 1$, and genes related to lipid uptake, such as $C D 36$, were upregulated upon HepG2 cells overexpressing uc.372 compared to control cells (Fig. 3a, b and Supplementary Fig. 3a). Similarly, overexpression of uc.372 in the livers of C57BL/6J mice led to elevated levels of acc, fas, scd1, and $\operatorname{cd} 36$ (Fig. 3c, d, and Supplementary Fig. 3b).

In contrast, suppression of uc.372 expression by transfection of Ad-uc.372i into HepG2 cells pre-treated with an $\mathrm{O} / \mathrm{P}$ mixture resulted in much lower mRNA and protein levels of ACC, FAS SCD1, and CD36 compared to control cells (Fig. 3e, f and Supplementary Fig. 3c). Moreover, silencing of uc.372 in the liver of HFD-fed mice reduced expression of acc, fas, scd1, and CD36 (Fig. 3g, h and Supplementary Fig. 3d). These results reveal that uc. 372 drives hepatic steatosis by promoting de novo lipogenesis and lipid uptake.

uc.372 inhibits the maturation of miR-195/miR-4668. To understand the mechanism by which uc.372 regulates the expression of ACC, FAS, SCD1, and CD36, we analyzed uc.372 distribution in HepG2 cells using in situ hybridization. In HepG2 cells, uc.372 was predominantly localized to the nucleus (Fig. 4a). The nuclear/cytoplasmic ratio of uc.372 expression in human HepG2 cells and Hep1-6 cells was 12.6 and 13.3, respectively (Fig. 4b), suggesting that uc.372 might exert its biological 
a

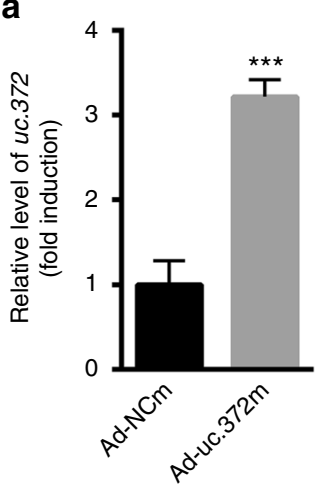

e
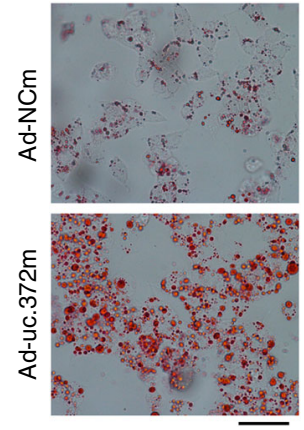

i

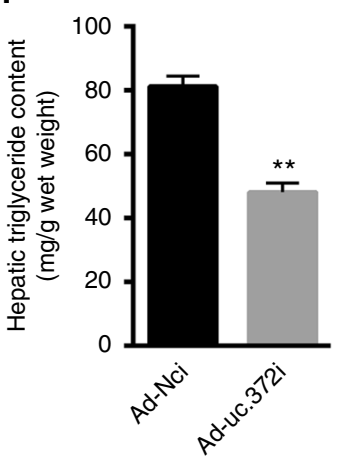

b

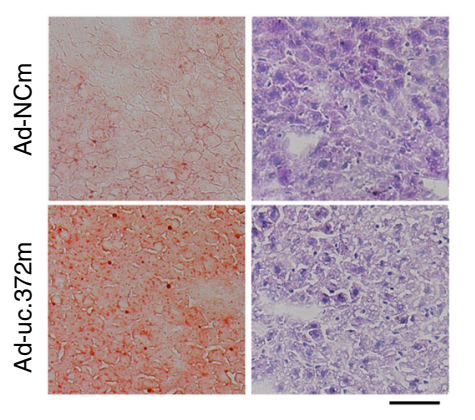

c

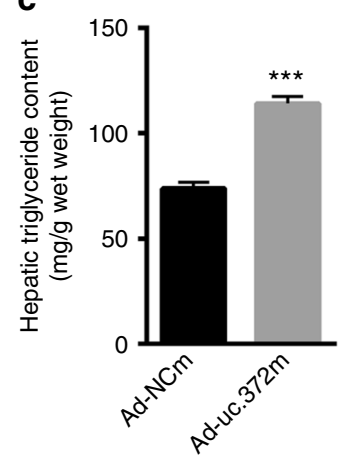

d

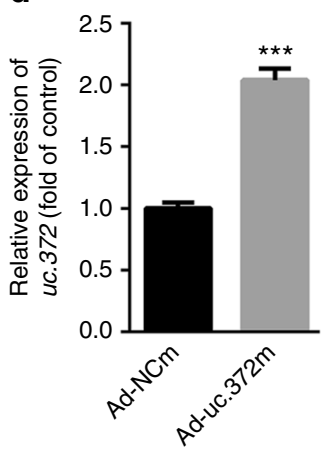

f

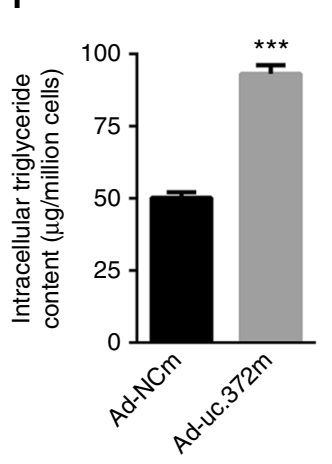

j

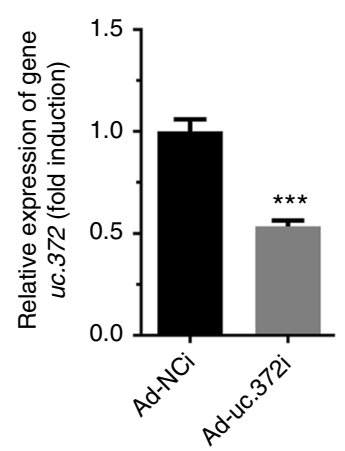

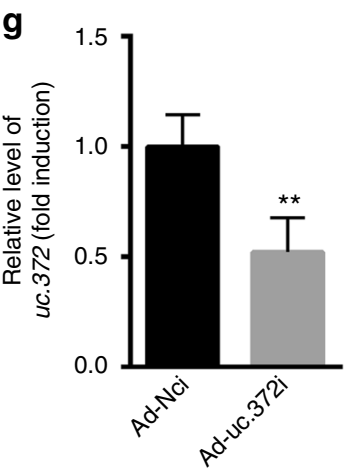

h

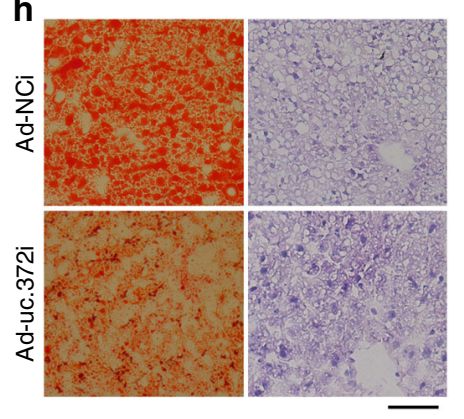

k

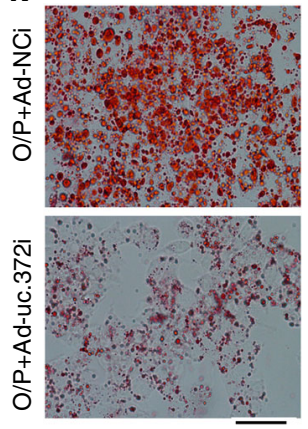

I

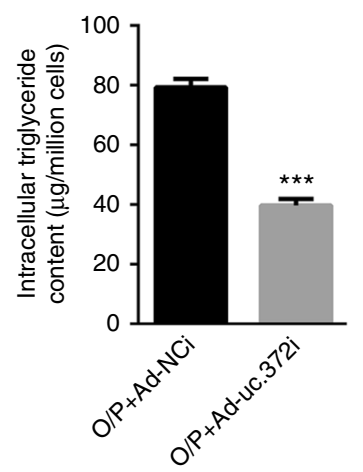

Fig. 2 Uc.372 drives hepatic lipid accumulation in mice. a Hepatic expression level of uc.372 in 6- to 8-week-old C57BL/6J mice administrated with Aduc. $372 \mathrm{~m}$ for 7 days by tail vein injection, as analyzed by real-time PCR $(n=5)$. b Representative image from three similar experiments of H\&E and Oil Red O staining in liver frozen sections of 6- to 8-week-old C57BL/6J mice after tail vein injection with Ad-uc.372 or Ad-NC for 7 days were shown. Scale bar, $400 \mu \mathrm{m}$. c Hepatic triglyceride content in 6- to 8-week-old C57BL/6J mice after tail vein injection with Ad-uc.372 or Ad-NC for 7 days $(n=5)$. d Expression level of uc.372 in HepG2 cells transfected with Ad-uc. $372 \mathrm{~m}$ or Ad-NC for $48 \mathrm{~h}(n=3)$. e Representative image from three similar experiments of Oil Red O staining in HepG2 cells transfected with Ad-uc.372m or Ad-NC for $48 \mathrm{~h}$. Scale bar, $40 \mu \mathrm{m}$. f Intracellular triglyceride content in HepG2 cells transfected with Ad-uc.372m or Ad-NC for $48 \mathrm{~h}(n=3)$. $\mathbf{g}$ Hepatic expression level of uc.372 in 10-week-HFD-fed mice administrated with Ad-uc.372i or Ad-NC for 7 days by tail vein injection $(n=5)$. h Representative image from three similar experiments of H\&E and Oil Red O staining in liver frozen sections of 10-week-HFD-fed mice after tail vein injection of Ad-uc.372i or Ad-NC for 7 days. Scale bar, $50 \mu \mathrm{m}$. i Hepatic triglyceride content in 10-week-HFD-fed mice after tail vein injection of Ad-uc.372i or Ad-NC for 7 days $(n=5)$. $\mathbf{j}$ Expression level of uc.372 in HepG2 cells transfected with Ad-uc.372i or Ad-NC for $48 \mathrm{~h}(n=3)$. k Representative image from three similar experiments of Oil Red O staining in HepG2 cells transfected with Ad-uc.372i or Ad-NC for 48 h. Scale bar, $40 \mu \mathrm{m}$. I Intracellular triglyceride content in HepG2 cells transfected with Ad-uc.372i or Ad-NC for $48 \mathrm{~h}(n=3)$. Data are mean \pm SEM; ${ }^{\star \star} P<$ $0.01 ;{ }^{\star \star \star} P<0.001$ vs. control group (a, $\mathbf{c}, \mathbf{g}, \mathbf{i}, \mathbf{j}, \mathbf{I}$ Student's $t$-test)

function in the nucleus. The correlation between miRNAs and UCEs has been widely reported ${ }^{20,24,25}$, indicating possible regulatory mechanisms that could connect both types of ncRNAs. To identify putative miRNA targets for uc.372, we overexpressed uc.372 in HepG2 cells and carried out a microarray analysis. A total of 66 miRNAs present in the array demonstrated $\geq 1.5$-fold upregulation or downregulation of expression upon uc.372 transfection (Fig. 4c, Supplementary Fig. 4a, 4b, and 4c). Of note, pri-miR-195 and pri-miR-4668 displayed a complementarity with the ultraconserved region of uc.372. This complementarity involved nucleotides located at the terminal loop region site within the miR-195 and miR-4668 primary transcripts (Fig. 4d). 

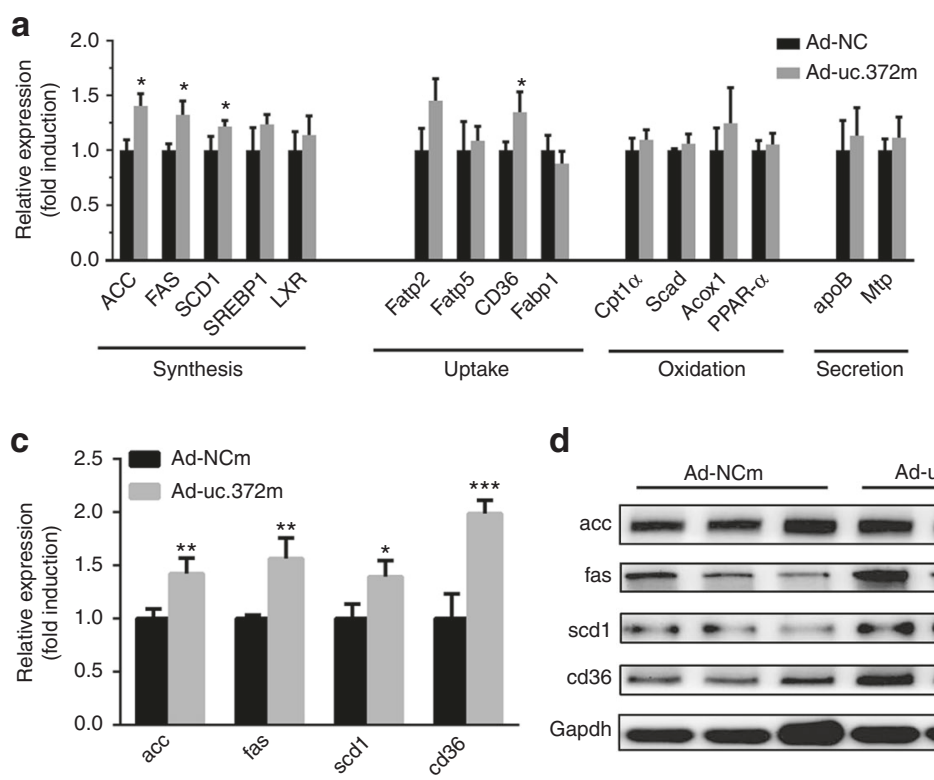

d
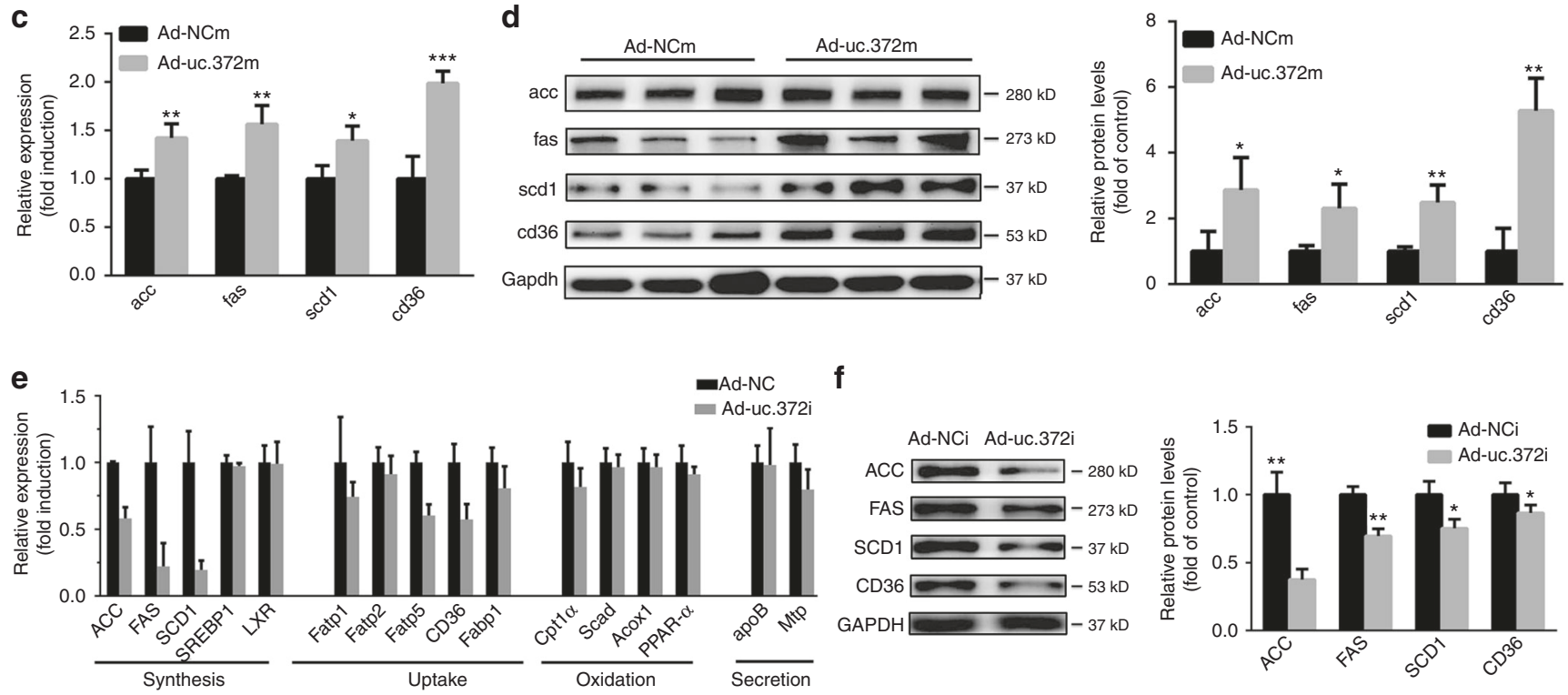

f
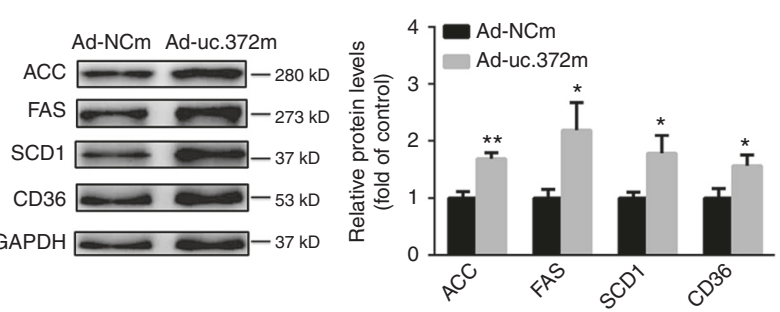

C

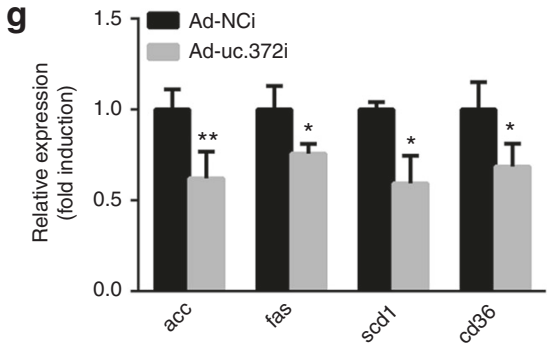

h
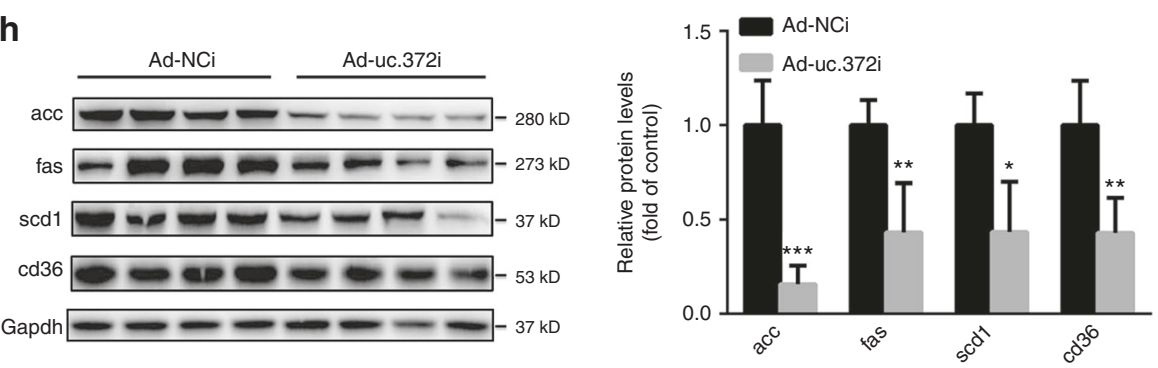

Fig. 3 Uc.372 promotes the expression of genes related to lipid synthesis and uptake in hepatocytes. a Expression levels of genes related to lipid synthesis (ACC, FAS, SCD, SREBP1, and LXR), uptake (Fatp1, Fatp2, Fatp5, Fabp1, and CD36), oxidation (Cpt1a, Scad, Acox1, and PPAR-a), and secretion (apoB and Mtp) in HepG2 cells transfected with Ad-uc. $372 \mathrm{~m}$ or Ad-NCm for $48 \mathrm{~h}(n=3)$. b Protein levels of ACC, FAS, SCD1, and CD36 in HepG2 cells transfected with Aduc.372m or Ad-NCm for $48 \mathrm{~h}$, as analyzed by western blot (representative blots from three similar experiments) $(n=3)$. c Expression levels of acc, fas, scd1, and $c d 36$ in the liver of 6- to 8-week-old C57BL/6J mice injected with Ad-uc.372m or Ad-NCm for 7 days $(n=3)$. d Protein levels of acc, fas, scd1, and cd36 in the liver of 6- to 8-week-old C57BL/6J mice injected with Ad-uc.372m or Ad-NCm for 7 days $(n=3)$. e Expression levels of genes related to lipid synthesis, uptake, oxidation, and secretion in HepG2 cells transfected with Ad-uc.372i or Ad-NCi for $48 \mathrm{~h}(n=3)$. f Protein levels of ACC, FAS, SCD1, and CD36 in HepG2 cells transfected with Ad-uc.372i or Ad-NCi for $48 \mathrm{~h}$ in the presence of an O/P mixture (representative blots from three similar experiments) ( $n=3)$. $\mathbf{g}$ Expression levels of acc, fas, scd1, and cd36 in the liver of 10-week-HFD-fed mice after tail vein injection of Ad-uc.372i or Ad-NC for 7 days $(n=3)$. h Protein levels of acc, fas, scd1, and cd36 in the liver of 10-week-HFD-fed mice after tail vein injection of Ad-uc.372i or Ad-NC for 7 days ( $n$ =3). Data are mean $\pm \mathrm{SEM} ;{ }^{\star} P<0.05 ;{ }^{\star \star} P<0.01 ;{ }^{\star \star \star} P<0.001$ vs. control group (Student's $t$-test)

In the nucleus, pri-miRNAs undergo Drosha-dependent cleavage into pre-miRNAs before being exported to the cytoplasm ${ }^{22}$. To explore the interaction between miR-195/miR-4668 and uc.372, we determined expression levels of pri-miR-195/pri-miR-4668, pre-miR-195/pre-miR-4668, and miR-195/miR-4668 upon uc.372 inhibition or overexpression. Of note, significantly reduced primiR-195/pri-miR-4668 and elevated pre-miR-195/pre-miR-4668 and mature miR-195/miR-4668 were observed in the HepG2 cells transfected with Ad-372i (Supplementary Fig. 5a). By contrast, overexpression of uc.372 in the HepG2 increased the levels of primiR-195/pri-miR-4668 and repressed the maturation of miR-195/ miR-4668 (Fig. 4e). To disrupt the interaction between uc.372 and these miRNAs, we constructed mutants in which the complementary nucleotides were deleted from uc.372 (Fig. 4d). As expected, when this mutant form of uc.372 was overexpressed, no changes in pri-miR-195/pri-miR-4668, pre-miR-195/pre-miR-4668, and 
a
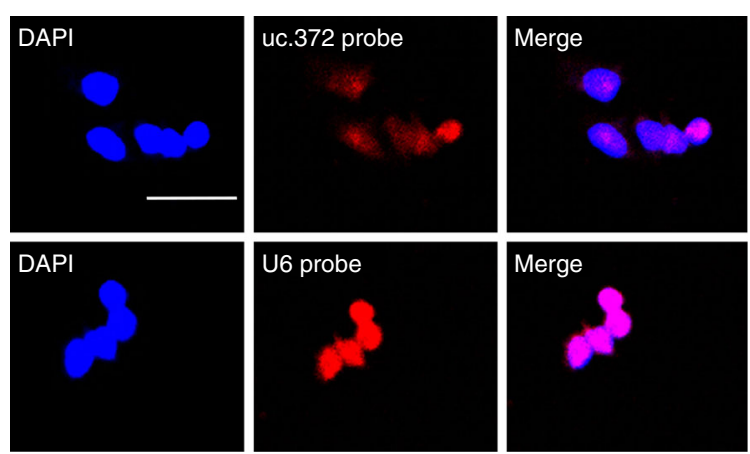

U6 probe

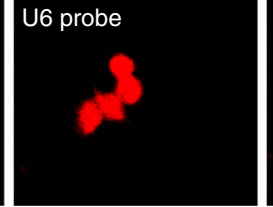

Merge

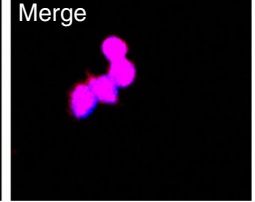

d

C b

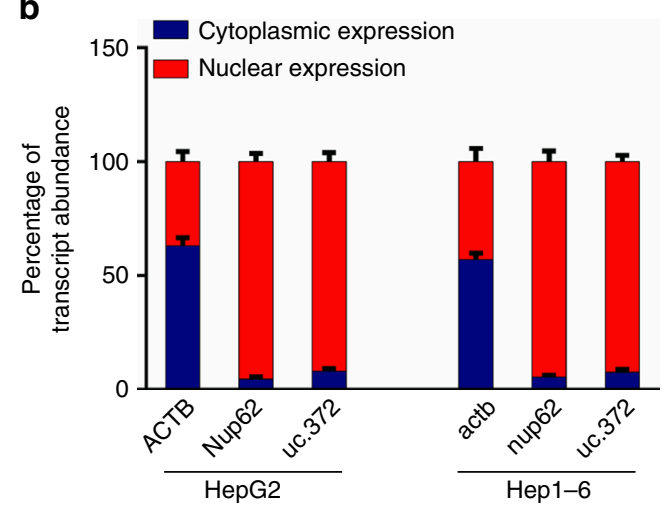

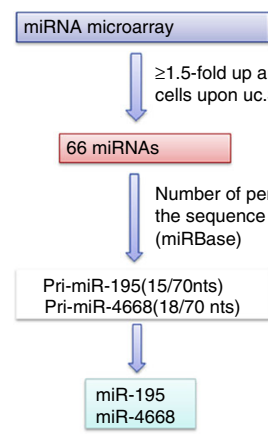

e
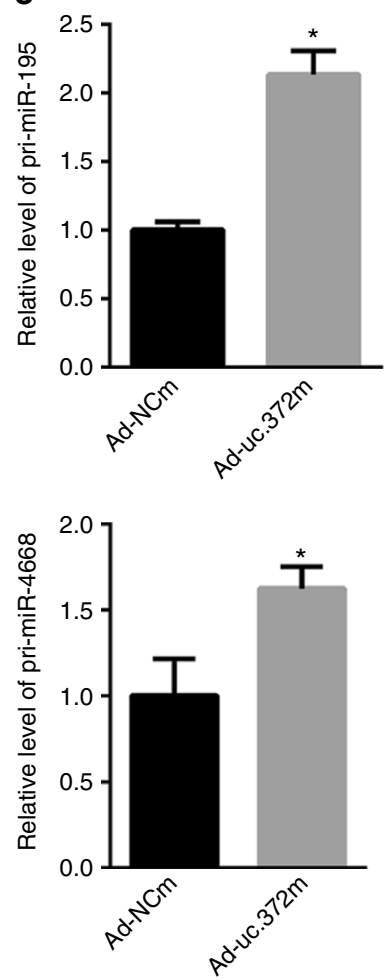
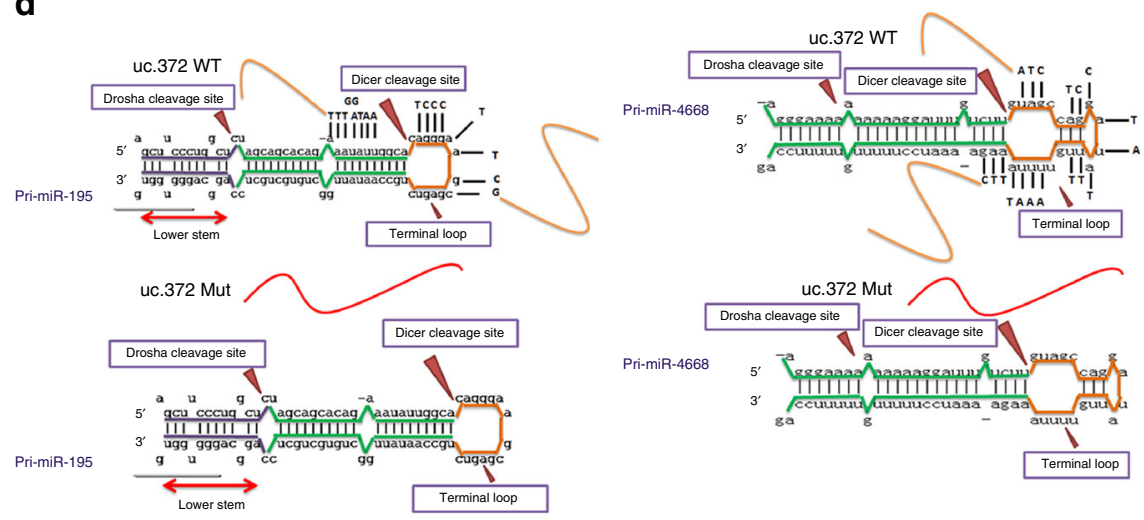
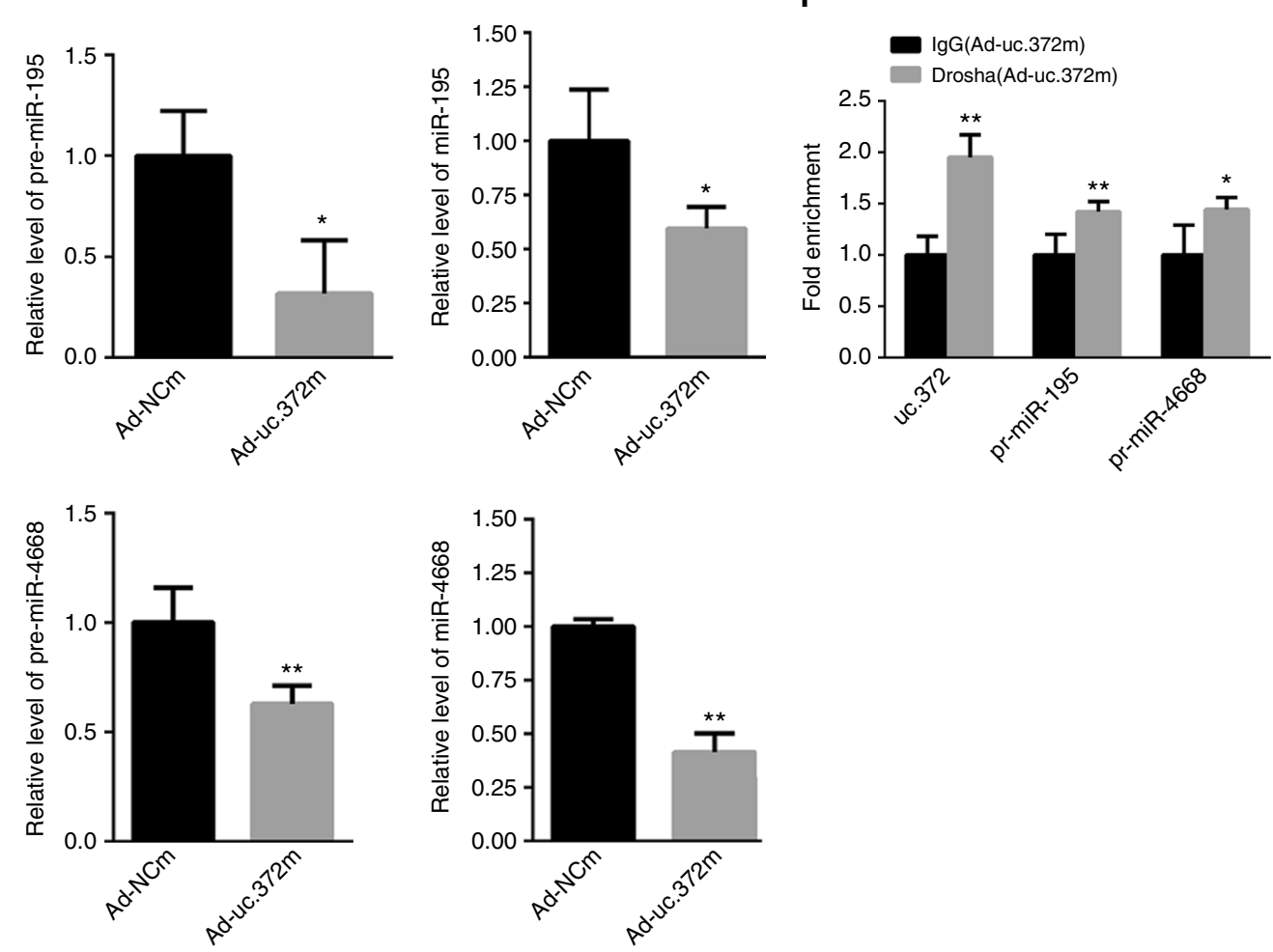

Fig. 4 uc.372 binds to pri-miR-195/pri-miR-4668. a Representative image from three similar experiments showed the distribution of uc.372 in HepG2 cells as analyzed by in situ hybridization. Scale bar, $25 \mu \mathrm{m}$. b Cellular fractionation assay in HepG2 and Hep1-6 cells was performed by quantitative real-time PCR using a specific cytosol control (gene ACTB) and a specific nuclear control (gene Nup62) $(n=3)$. c Schematic flowchart depicting the strategy. $\mathbf{d}$ The stem-loop sequence of pri-miR-195 (left panel) and pri-miR-4668 (right panel), and their partial complementarity with uc.372. e The levels of pri-miR-195/ pri-miR-4668, pre-miR-195/pre-miR-4668, and mature miR-195/miR-4668 in the HepG2 cells transfected with Ad-uc.372m or Ad-NCm for 48 h ( $n=3$ ). f RIP assay showed that uc.372 could bind pri-miR-195/pri-miR-4668 in HepG2 cells transfected with Ad-uc.372m or Ad-NCm for 48 h ( $n=3$ ). Data are mean $\pm \mathrm{SEM} ;{ }^{\star} P<0.05 ;{ }^{\star \star} P<0.01$ vs. control group (Student's $t$-test) 
mature miR-195/miR-4668 expression were identified (Supplementary Fig. 5b). To further assess that pri-miR-195/pri-miR-4668 interacts with uc.372, RNA-binding protein immunoprecipitation (RIP) assay was performed with an antibody against Drosha, a key protein in the processing of pri-miRNA into pre-miRNA. As shown in Fig. 4f, overexpression of uc.372 significantly increased the level of pri-miR-195/pri-miR-4668 in the immunoprecipitated RNA with antibody against Drosha, indicating a binding between uc.372 and the terminal loop region of pri-miR-195/pri-miR-4668.

Given the direct RNA-RNA interaction, we assessed whether uc.372 affected lipid metabolism through miR-195/miR-4668mediated target genes. Previous studies have shown that ACC and FAS are target genes of miR- $195^{26}$ (Supplementary Fig. 6a). Based on the reduced levels of miR-195 in HepG2 cells treated with an $\mathrm{O} / \mathrm{P}$ mixture (Supplementary Fig. 6a), we investigated the effects of miR-195 on expression of ACC and FAS. As shown by realtime PCR analysis, miR-195 markedly suppressed mRNA levels of FAS and ACC, even when uc.372 was overexpressed in HepG2 cells (Fig. 5a, b). More importantly, the uc.372 inhibitiondependent reduction in ACC and FAS expression and impaired intracellular triglyceride contents could be largely restored by miR-195 suppression (Fig. 5c, Supplementary Fig. 6b, Supplementary Fig. 7a, b), suggesting that reduced miR-195 suppression of ACC and FAS expression plays a key role in uc.372-induced lipid accumulation.

Based on TargetScan, SCD1 and CD36 were predicted as possible target genes of miR-4668 (Supplementary Fig. 6c). We determined expression of miR-4668 in HepG2 cells treated with an $\mathrm{O} / \mathrm{P}$ mixture, and observed decreased expression of miR-4668 in the $\mathrm{O} / \mathrm{P}$-treated HepG2 cells compared to controls (Supplementary Fig. 6c). To verify that SCD1 and CD36 are true targets of miR-4668, the 3 ' untranslated regions (UTRs) of these genes containing the binding sites for miR-4668 were cloned into the pmirGLO plasmid to assess luciferase activity. As shown in Fig. 5d, miR-4668 significantly suppressed the relative luciferase units of pmirGLO-SCD1-3'UTR or pmirGLO-CD36-3'UTR, whereas these affects were rescued by expression of pmirGLOSCD1-3'UTR pmirGLO-CD36-3'UTR mutants lacking the miR4668-binding site. Further studies revealed that miR-4668 reduced the mRNA levels of SCD1 and CD36 even when uc.372 was overexpressed in HepG2 cells (Fig. 5e, f). Conversely, the uc.372 silencing-dependent reduction in SCD1 and CD36 expression and lipid accumulation could be partially rescued by miR-4668 inhibition (Fig. 5g, Supplementary Fig. 6d, Supplementary Fig. 7c, d).

Taken together, these data demonstrate that uc.372 specifically suppresses miR-195/miR-4668 expression by binding to pri-miR195/pri-miR-4668 to relieve miR-195/miR-4668-mediated suppression of functional target genes such as ACC, FAS, SCD1, and $C D 36$, leading to lipid accumulation in hepatocytes.

INSM2 may be involved in the transcription of uc.372. We next sought to understand how the transcription of uc. 372 is regulated. By analyzing the sequence of uc.372, we found that it consists of 277 nucleotides (nt) that are highly conserved across species. The uc.372 ultraconserved region is located within the intronic region of the RALGAPA1 gene and is also located $1400 \mathrm{bp}$ downstream of the insulinoma-associated 2 gene (INSM2) on chromosome 14 (Fig. 6a). To explore the possible interrelationships between RALGAPA1, INSM2, and uc.372 transcription, the mRNA levels of RALGAPA1 and INSM 2 were first evaluated by real-time PCR in the livers of $\mathrm{db} / \mathrm{db}$ mice and HFD-fed mice. The results indicated that the expression of both RALGAPA1 and INSM2 was increased in the livers of these animal models (Fig. 6b, c). In HepG2 cells treated with a $300 \mu \mathrm{M}$ O/P mixture, the mRNA levels of RALGAPA1 and INSM2 were also enhanced (Fig. 6d, e). Furthermore, no changes in uc.372 expression were identified in HepG2 cells transfected with siRNA against RALGAPA1, despite a $71-78 \%$ decrease in RALGAPA1 mRNA levels (Fig. 6f). However, uc.372 expression was significantly downregulated in HepG2 cells treated with siRNAs targeting INSM2 (Fig. 6g). These observations suggest that INSM2 may be involved in the transcriptional regulation of uc.372.

USF1 transcriptionally regulates INSM2 and uc.372 expression. Given that transcription of uc.372 is dependent on INSM2, we proceeded to explore the transcription factors modulating this process. Six transcription factors targeting uc.372 were identified through the Gene Regulation database (http://www.gene-regulation.com/pub/databases.html). As shown by real-time PCR analysis, the expression of upstream transcription factor 1 (USF1) was elevated in the livers of both $\mathrm{db} / \mathrm{db}$ and HFD-fed mice (Fig. 7a, b). We also observed a significantly increased level of USF1 in HepG2 cells treated with an $\mathrm{O} / \mathrm{P}$ mixture (Fig. 7c). The consistent expression patterns of USF1 with INSM2 raised the possibility that USF1 could transcriptionally regulate expression of INSM2. To further test this hypothesis, USF1 was overexpressed in HepG2 cells by adenovirus transduction. Compared to control cells, cells overexpressing USF1 showed markedly enhanced mRNA levels of uc.372 and INSM2, but not the negative control of NOX2 (Fig. 7d). In addition, overexpression of USF1 also resulted in a significant increase in the protein levels of ACC, FAS, SCD1, and CD36 (Fig. 7e, Supplementary Fig. 8a). In contrast, decreasing USF1 expression reduced the mRNA levels of uc.372 and INSM2, but not the negative control of NOX2 (Fig. 7f) and the protein levels of ACC, FAS, SCD1, and CD36 (Fig. 7g, Supplementary Fig. 8b). Importantly, overexpression of USF1 failed to enhance uc.372 expression in HepG2 cells with knockdown of INSM2 (Fig. 7h), indicating that direct or indirect regulation of INSM2 on uc.372 may exist since INSM2 is a well-characterized transcriptional repressor $2^{27}$. To further verify that USF1 exerts its functional role through regulation of uc.372, we employed a rescue strategy in HepG2 cells. Inhibition of uc.372 significantly blunted the USF1-mediated induction of $A C C, F A S, S C D 1$, and CD36 expression (Fig. 7I, j). Based on these data, we propose that USF1 transcriptionally regulates the expression of INSM2 and uc.372, thereby elevating the expression of $A C C, F A S, S C D 1$, and CD36 in hepatocytes.

The effect of uc.372 was validated in NAFLD patients' livers. We next asked whether uc.372 was functionally involved in the abnormal hepatic lipid accumulation of NAFLD patients by regulation of pri-miR-195/pri-miR-4668 processing. In addition to abnormal lipid accumulation, we also observed a marked upregulation of hepatic uc.372 expression in NAFLD patients (Fig. 8a, b). Microarray transcriptome profiling analysis revealed that lipid metabolism pathways and uc.372 expression were aberrantly changed in the liver of NAFLD patients (Supplementary Fig. 9a, b). This prompted us to further examine genes related to lipogenesis and lipid uptake using real-time PCR. We confirmed that the levels of ACC, FAS, SCD1, and CD36 were significantly elevated in the liver of NAFLD patients (Fig. 8c). Congruently, we found significant enhancement of hepatic INSM2 and USF1 expression, supporting the idea that USF1 transcriptionally regulates INSM2 and uc.372 expression in the liver of NAFLD patients (Fig. 8d, e). Subsequently, we analyzed the hepatic levels of pri-miR-195/pri-miR-4668 and mature miR$195 / \mathrm{miR}-4668$ in these patients. Intriguingly, we observed an 
a

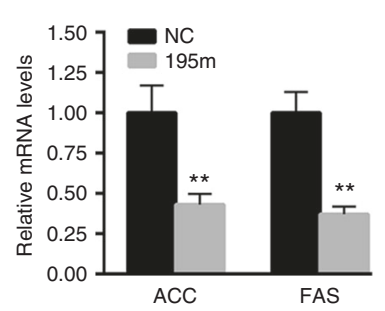

C

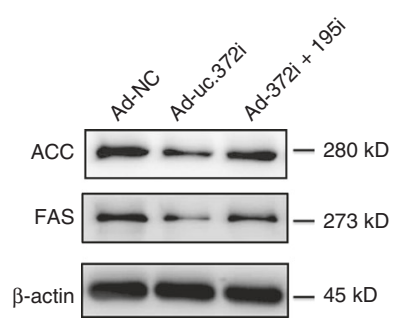

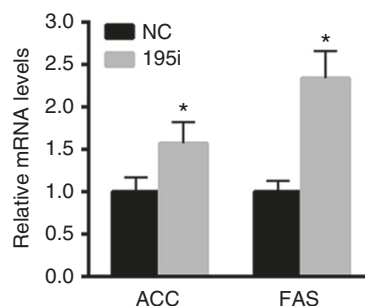

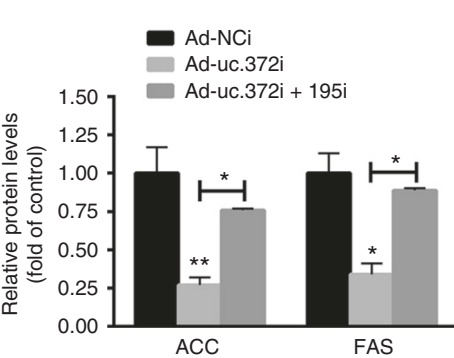

b
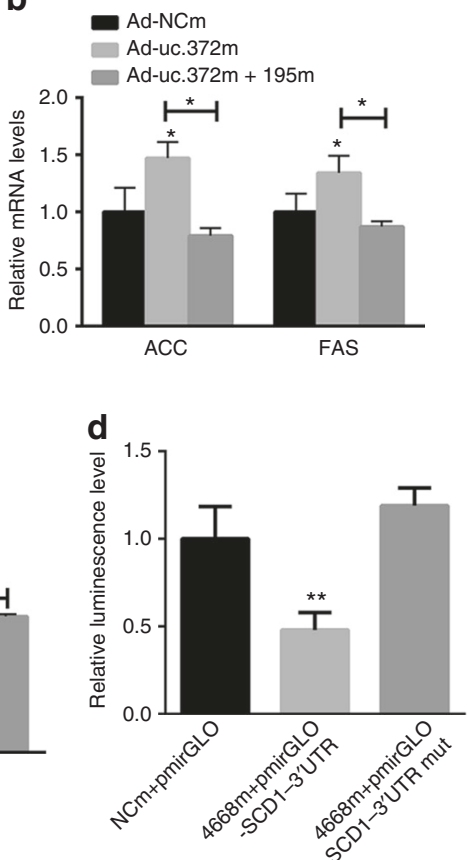

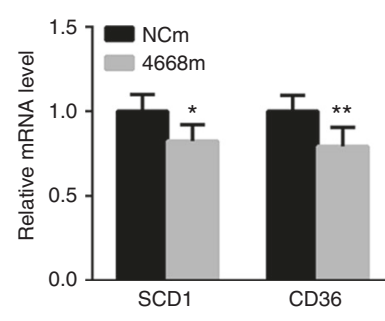

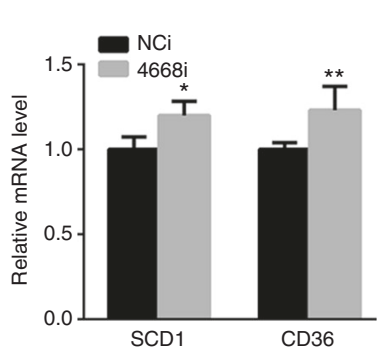

\section{f}

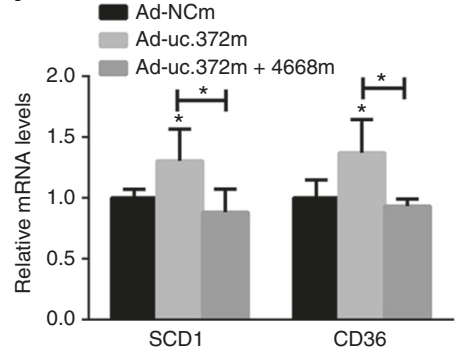

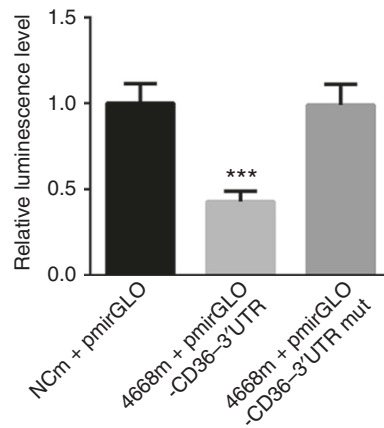
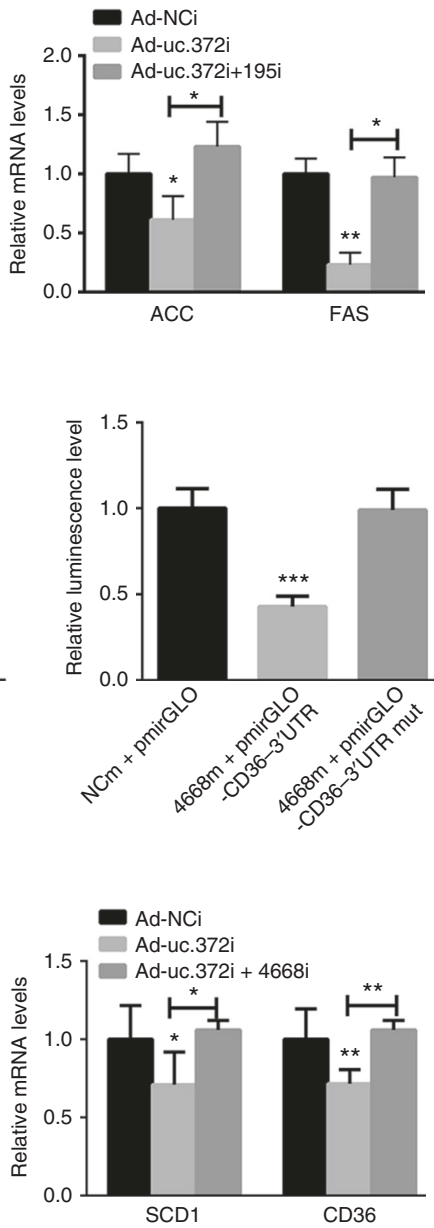

g
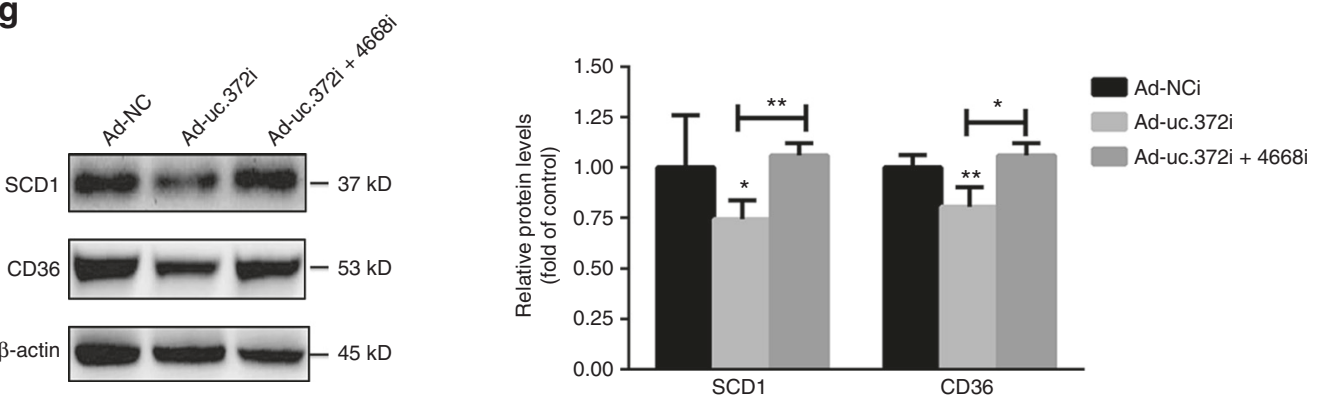

Fig. 5 uc.372 inhibits the maturation of miR-195/miR-4668 to regulate expression of ACC, FAS, SCD1, and CD36. a Expression levels of ACC and FAS in the HepG2 cells transfected with miR-195 mimic and inhibitor at a final concentration of $20 \mathrm{nM}$ for $48 \mathrm{~h}(n=3)$. $\mathbf{b}$ Expression levels of $A C C$ and FAS in the HepG2 cells transfected with miR-195 mimic and inhibitor for $24 \mathrm{~h}$ in the presence with Ad-uc.372m and Ad-uc.372i $(n=3)$. c Protein levels of ACC and FAS in Ad-uc.372i-infected and miR-195 inhibitor-transfected HepG2 cells in the presence with with $300 \mu \mathrm{M} \mathrm{O/P}$ mixture for $48 \mathrm{~h}$ (representative blots from three similar experiments) $(n=3)$. d The relative luciferase units (RLU) in the HepG2 cells transfected with pmirGLO-SCD1-3'UTR and pmirGLOSCD1-3'UTR mutant or pmirGLO-CD36-3'UTR and pmirGLO-CD36-3'UTR mutant under overexpression of miR-4668 or NC for $48 \mathrm{~h}(n=3$ ). e Expression levels of SCD1 and CD36 in the HepG2 cells transfected with miR-4668 mimic and inhibitor or NC for $48 \mathrm{~h}(n=3)$. $\mathbf{f}$ Expression levels of SCD1 and CD36 in the HepG2 cells transfected with miR-4668 mimic and inhibitor for $24 \mathrm{~h}$ in the presence with Ad-uc.372m and Ad-uc.372i $(n=3)$. $\mathbf{g}$ Protein levels of ACC and FAS in Ad-uc.372i-infected and miR-4668 inhibitor-transfected HepG2 cells in the presence with $300 \mu \mathrm{M} \mathrm{O/P}$ mixture for $48 \mathrm{~h}$ (representative blots from three similar experiments) $(n=3)$. Data are mean $\pm \mathrm{SEM} ;{ }^{\star} P<0.05 ;{ }^{\star \star} P<0.01 ;{ }^{\star \star \star} P<0.001$ vs. control group (a, e Student's $t$-test; $\mathbf{b}, \mathbf{c}$, $\mathbf{d}, \mathbf{f}, \mathbf{g}$ analysis of variance (ANOVA))

increase of pri-miR-195/pri-miR-4668 and a reduction of miR-195/miR-4668 expression in the liver of NAFLD patients (Fig. 8f, g). These results confirm that the lipid accumulation effect of uc.372 is subject to regulation by miR-195/miR-4668 in the steatotic liver of NAFLD patients.

\section{Discussion}

Recently, ucRNAs have been identified as a class of highly conserved lncRNAs, but a clear understanding of the physiological significance and potential pathological roles of ucRNAs has remained elusive. Much of the research on ucRNAs has focused 
a

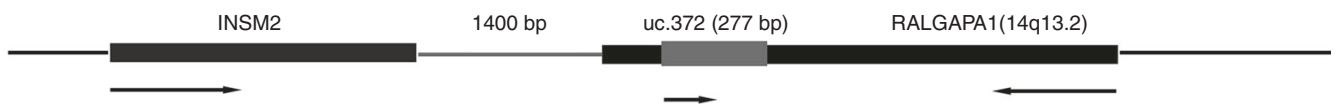

b

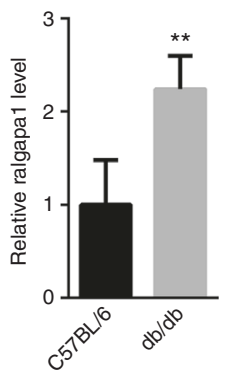

$\mathbf{f}$

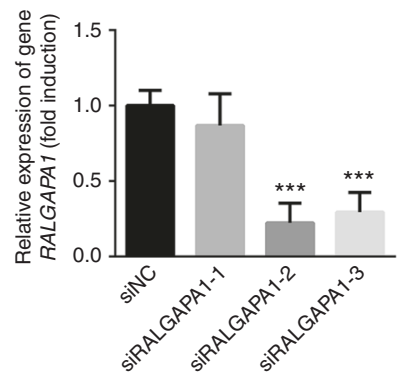

C
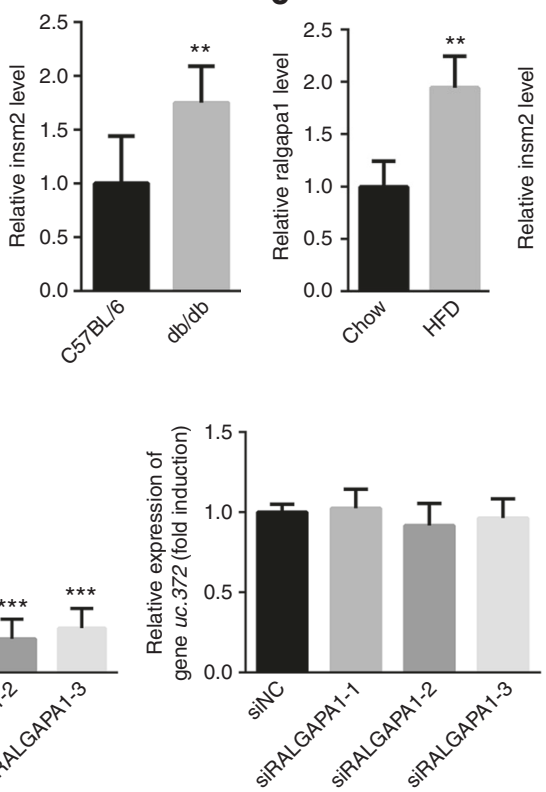

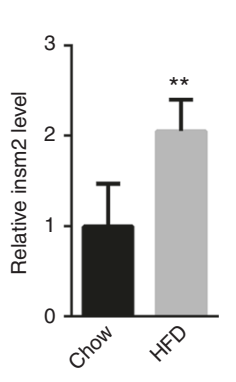

d e

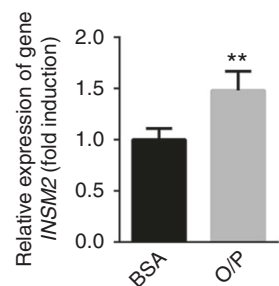

g

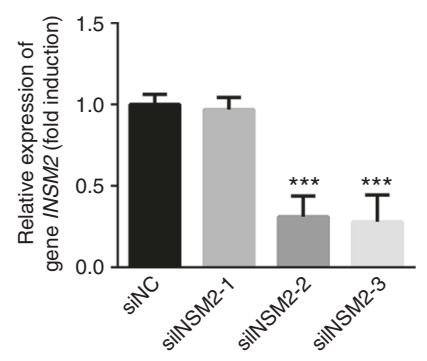

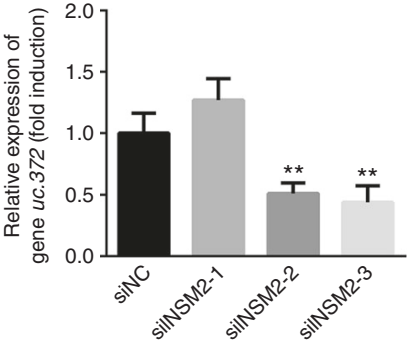

Fig. 6 uc.372 expression is dependent on transcription of INSM2. a Schematic analysis of uc.372 location. b Ralgapa1 and insm2 expression in the liver of 8 week-old male $\mathrm{db} / \mathrm{db}$ mice $(n=5)$. c Ralgapa1 and insm2 expression in the liver of 10-week-HFD-fed mice ( $n=5$ ). $\mathbf{d}$ The mRNA level of RALGAPA1 in the HepG2 cells treated with $300 \mu \mathrm{M}$ O/P mixture for $48 \mathrm{~h}(n=3)$. e The mRNA level of INSM2 in the HepG2 cells treated with $300 \mu \mathrm{M}$ O/P mixture for $48 \mathrm{~h}$ $(n=3)$. $\mathbf{f}$ The levels of RALGAPA1 and uc.372 after silencing RALGAPA1 in HepG 2 cells for $48 \mathrm{~h}(n=3)$. $\mathbf{g}$ The levels of INSM2 and uc.372 after silencing

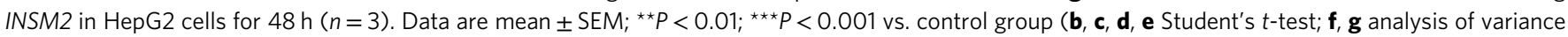
(ANOVA))

on elucidating their evolutionary significance 28,29 and the tumor oncogenic or suppressive roles of these ucRNAs in human cancers $^{20,21,23}$. Moreover, emerging reports indicate that lncRNAs are key regulators of lipid metabolism and fatty liver disease $^{30,31}$. For example, lncLSTR, a liver-enriched lncRNA, regulates a TDP-43/FXR/ApoC2-dependent pathway to maintain lipid metabolism in mice ${ }^{32}$. However, the role of ucRNAs in lipid metabolism and pathogenesis of NAFLD remained unclear. In the present study, we identify the upregulation of uc.372 in the livers of $\mathrm{db} / \mathrm{db}$ and HFD-fed mice and NAFLD patients. It is very interesting that the expression of uc.348, 372, 94, 157, and 436 was dramatically increased in the liver of $\mathrm{db} / \mathrm{db}$ mice, but not in C57 mice at 10 weeks after HFD administration. To analyze the reason for this inconsistence, we further examined expression of uc. $348,372,94,157$, and 436 in the liver of C57 mice at 5, 10, 15, and 22 weeks after HFD administration and the liver of NAFLD patients. The level of uc.372 was continuously increased in the liver of C57 mice at 5, 10, 15, and 22 weeks, whereas the levels of uc.348 and 94 were increased at 15 or 22 weeks after HFD administration. However, no changes of uc.157 and 436 were identified (Supplementary Fig. 1d). Moreover, in the liver of NAFLD patients, the expression levels of uc.372 and uc.157 were increased, but that of uc.94 was decreased (Supplementary Fig. 9c). These results indicated that this inconsistence might be due to different pathogeneses or pathological stages. The liver is well established as the most important organ for the biogenesis of major metabolites such as lipids ${ }^{33}$. In the liver, aberrant hepatic fat deposits result in the development of NAFLD ${ }^{34}$. Our data demonstrate an important contribution of hepatic uc.372 to the impaired homeostasis of lipid metabolism and implicate it as a previously uncharacterized lncRNA that plays a critical role in the pathogenesis of NAFLD. It is noteworthy that in the HFD-fed mice, the expression of uc.372 was dramatically increased in the muscle and fat, the organs also closely participating in NAFLD progression due to their critical roles in lipid metabolism and insulin resistance. To assess whether the regulation of hepatic steatosis by uc. 372 is a direct effect on hepatocytes or derives from its influence on fat or muscle, for instance, the lipolysis of adipocytes, we examined the expression of green fluorescent protein (GFP) and uc.372 in different tissues after tail vein injection with Ad-uc.372m. After injection, GFP was mainly expressed in the liver of mice (Supplementary Fig. 2g). Similarly, Ad-uc.372m injection led to dramatically increased uc.372 in the liver, but not in other tissues (Supplementary Fig. 2h). These data suggest that overexpressed hepatic uc.372 directly induced hepatic steatosis. So, what is the effect of uc.372 on the lipid metabolism in adipose tissue and muscle? Given the importance of skeletal muscle to our overall physical and metabolic capacities $^{35}$, improving our understanding of the changes to muscle health during the development of obese and insulin resistant states will advance the development of therapeutic strategies for those individuals. While the exact cause of muscle metabolism disorders with the development of diet-induced obesity is still unknown, one proposed mechanism is elevated delivery of free fatty acids (FFAs) in conjunction with impaired lipid metabolism within skeletal muscle leading to a build-up of intramyocellular lipids and associated lipid derivatives ${ }^{36}$. Recent advances have shown that IncRNAs are associated with skeletal muscle 
development, growth, and function ${ }^{37-39}$. For instance, H19, malat1, MyoD upstream lncRNA, lncMyoD, developmental pluripotency-associated 2 upstream binding muscle lncRNA, and Linc-MD $1^{37-40}$ are all regulated during myogenesis, and likely necessary for efficient differentiation. However, little study has focused on the role of lncRNAs in the lipid metabolism of muscle tissues in HFD mice. In the present study, we found the level of uc. 372 was increased in the muscle tissues of HFD mice compared with that of control. Moreover, pre-incubation with $\mathrm{O} / \mathrm{P}$ significantly enhanced the level of uc.372 in the liver cells. a

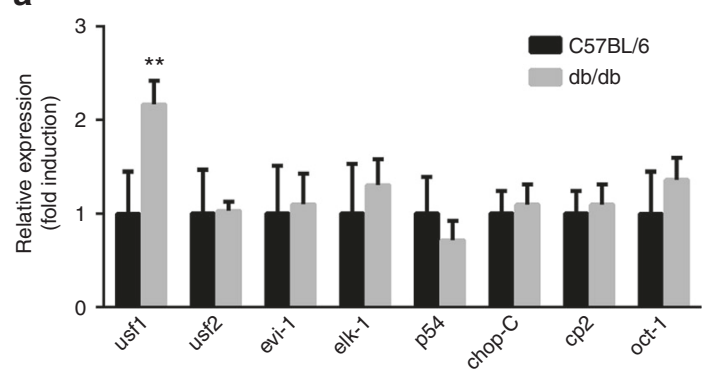

b

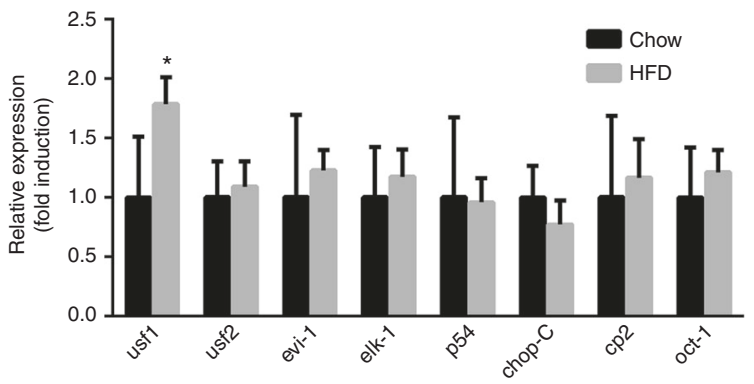

C

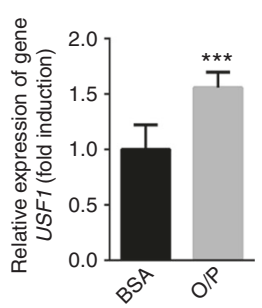

d

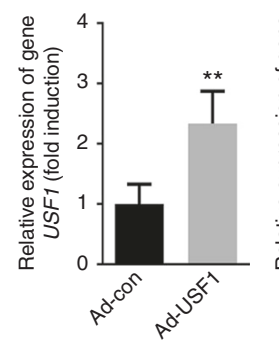

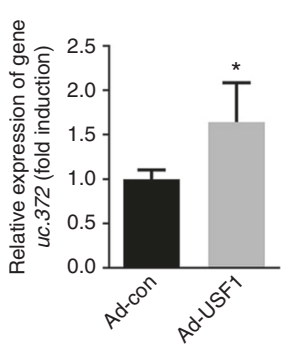

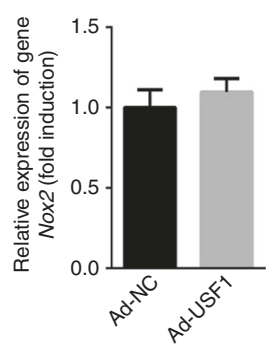

e

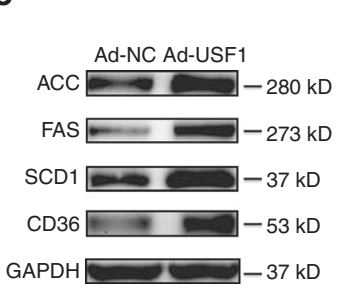

g

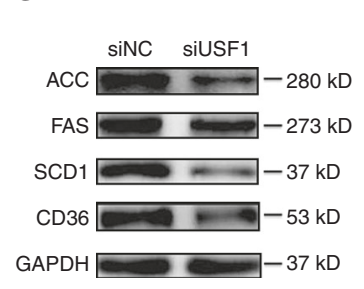

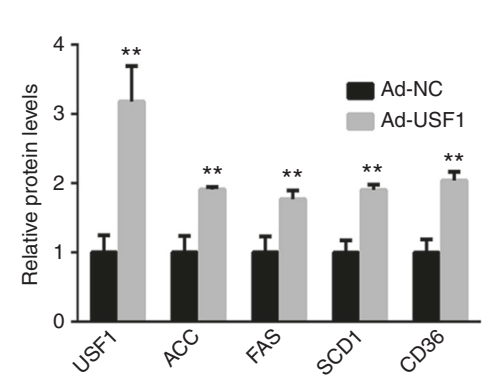

f
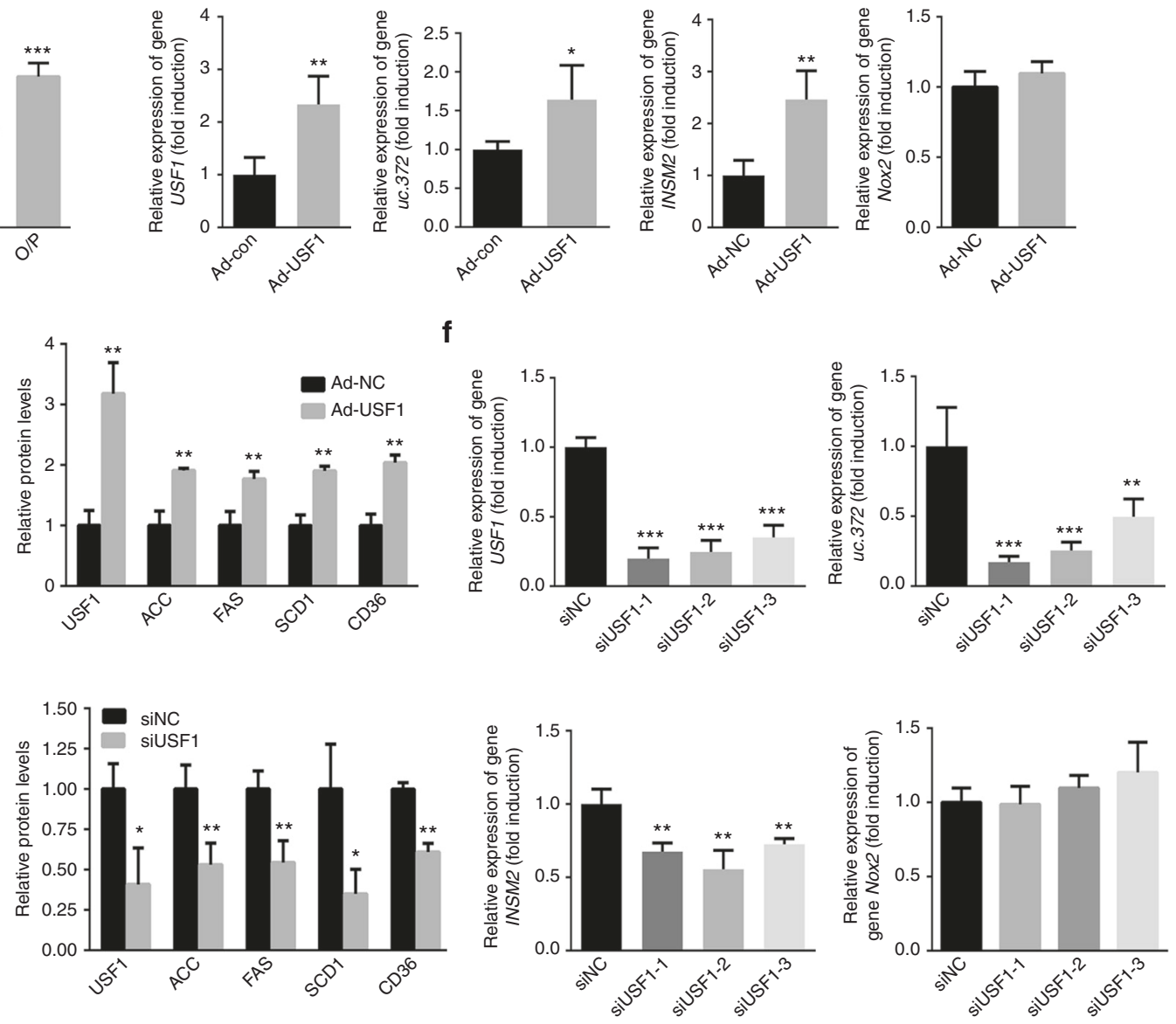

h

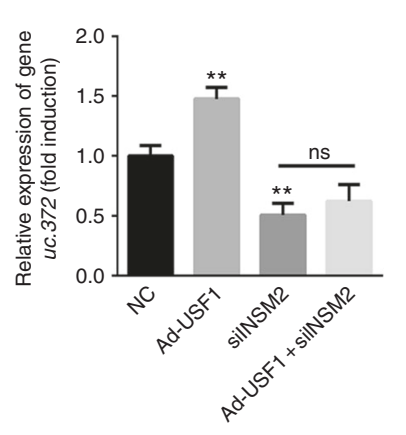

i

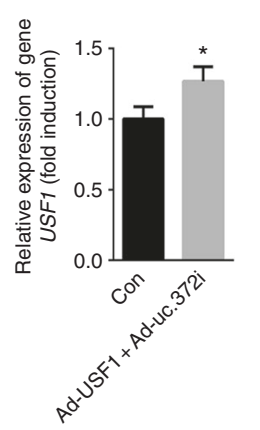

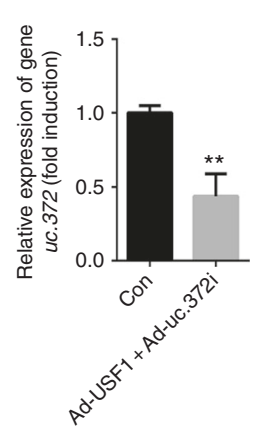

j

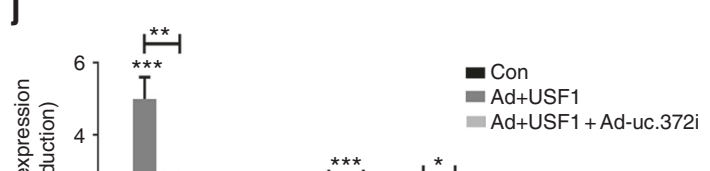


Therefore, we propose that the elevated FFAs level in muscle may induce the expression of uc.372, which is then involved in the progression of HFD-induced metabolic disorders. In the future, it is necessary to explore the role of uc.372 in fat or muscle, for instance, the lipolysis of adipocytes, by using fat or musclespecific uc.372-TG and $\mathrm{KO}$ mice.

It has been demonstrated that multiple factors are involved in hepatic fat accumulation, including enhanced de novo lipogenesis and absorption as well as decreased fatty acid oxidation and secretion ${ }^{41}$. To provide further insights to the role of uc. 372 in hepatic lipid metabolism, we analyzed the expression of genes related to lipid metabolism in vivo and in vitro. We found that uc.372 regulated expression of genes related to lipid synthesis, including $A C C, F A S$, and $S C D 1$, and genes related to lipid uptake, such as $C D 36$, suggesting that uc.372 drives hepatic lipid accumulation through the promotion of de novo lipogenesis and lipid uptake.

We next sought to understand how uc.372 regulates expression of ACC, FAS, SCD1, and CD36. Of note, intronic ultraconserved regions are frequently related to the regulation of transcription and DNA binding, indicating a potential role as regulators of gene expression ${ }^{16}$. Given the structural similarity of miRNAs and lncRNAs, miRNAs may also bind to lncRNA sequences. For instance, multiple lncRNAs have been identified as competing endogenous RNAs (ceRNAs) ${ }^{42}$. In general, ceRNAs function as miRNA sponges and decrease the activity of target miRNAs without changing their biogenesis ${ }^{43,44}$. Recent studies have demonstrated IncRNA-mediated regulatory networks for miRNA processing $^{20,22}$. For example, lower-stem strand invasion by uc. $283+$ A impairs microprocessor recognition and efficient primiR-195 cropping ${ }^{22}$. The processing of pri-miRNA into premiRNA requires direct interaction with the Drosha-DGCR8 microprocessor complex to modulate miRNA maturation ${ }^{45,46}$. The pri-miRNA/pre-miRNA hairpin structure consists of mismatches, internal loops, and bulges ${ }^{47}$. The terminal loop of these hairpins demonstrates a variable structure, which may be important for the rate at which a specific miRNA is processed ${ }^{48}$. It is suggested that a flexible terminal loop plays a key role in processing by Drosha, as decreasing the length or changing the sequence of a terminal loop significantly influences pri-miRNA processing efficiency ${ }^{49,50}$. In situ hybridization and cytoplasmic and nuclear fractioning demonstrates that uc.372 is mainly located in the nucleus of HepG2 cells. The nuclear localization of uc.372 suggests it may bind to mRNA to modulate gene transcription. Further studies by RIP assay revealed that pri-miR-195 and pri-miR-4668 interacts with the ultraconserved region of uc.372 at the terminal loop region site within the miR-195 and miR-4668 primary transcript. More importantly, uc.372 regulates pri-to-pre-miRNA cleavage of miR-195 and 4668 by binding primiR-195/pri-miR-4668.

Abnormal expression of miR-195 has been widely reported in diverse biological systems ${ }^{51,52}$. For instance, miR-195 can bind the 3 'UTRs of cyclin D1,CDK6, and E2F3, thereby suppressing cell proliferation in human hepatocellular carcinoma cells ${ }^{52}$. Additionally, miR-195 suppresses cell proliferation, invasion, and metastasis in breast cancer cells by targeting FAS, HMGCR, ACC, and $C Y P 27 B 1^{26}$. Consistently, we found that miR-195 markedly suppresses mRNA levels of FAS and ACC, even when uc.372 is overexpressed in HepG2 cells. However, less is known about the functional role of miR-4668. Our results suggest that uc.372 suppresses miR-4668 expression through binding pri-miR4668 to relieve miR-4668-mediated suppression of functional target genes such as SCD1 and CD36.

How miR-195/miR-4668 link to the metabolic genes such as FAS, ACC, SCD1, and CD36? It was showed that once miRNAs processed from the hairpin and loaded into the Ago2 of the silencing complex, the miRNAs pair with mRNAs to direct posttranscriptional repression ${ }^{53}$. In animals, the canonical model posits that the 22- to 23-nt miRNAs guide Ago2 to target sites in mRNA 3'UTR and coding sequences through imperfect sequence complementarity. Complementarity in the "seed" region of the miRNA, nt 2-8, is a key determinant of the interaction ${ }^{54}$. At sites with extensive pairing complementarity, metazoan miRNA can direct Argonaute-catalyzed mRNA cleavage ${ }^{53,55}$. In the present study, the sequence alignment results in Supplementary Fig. 6a, b showed canonical complementarity between miR-195/miR-4668 and their respective targets, which is necessary for the formation of the miRNA-mRNA duplex ${ }^{56}$. And dual luciferase reporter assay suggested that miR-195/miR-4668 could reduce the relative luciferase activity. Based on the previous studies and our observations, we consider that $F A S$ and $A C C / S C D 1$ and $C D 36$ are direct targets of miR-195/miR-4668 mediated by Ago2.

Finally, we identify USF1 as transcription factor that regulates uc.372 expression. Through the Gene Regulation database, we found that the sequence of uc.372 overlaps with that of $R A L$ GAPA1 and is located downstream of INSM2, which plays a key role in insulin islet development ${ }^{27}$. Further studies revealed that the transcription of RALGAPA1 and uc.372 is independent. However, our data showed that knockdown of INSM2 suppressed the level of uc.372 and the regulation of USF1 on uc.372. How INSM2 mediates regulation of USF1 on uc.372? If uc.372 shares the promoter with INSM2, the regulation on each should be independent of the other. We propose that direct or indirect regulation of INSM2 on uc.372 may exist since INSM2 is a wellcharacterized transcriptional repressor $2^{27}$. Therefore, further study is necessary to elucidate the potential mechanism underlying the regulation of uc.372. In the present study, we identify USF1, which has been shown to participate in the transcriptional regulation of several lipid metabolism-related genes ${ }^{57,58}$, as a major transcription factor regulating the expression of INSM2 and uc.372, thereby mediating uc.372-induced hepatic lipid accumulation.

In summary, as shown in Fig. 8h, our findings reveal a novel mechanism by which uc.372 drives hepatic steatosis by repressing

Fig. 7 Upstream transcription factor 1 (USF1) transcriptionally regulates expression of INSM2 and uc.372. a Identification of usf1 elevated in the liver of 8 week-old male $\mathrm{db} / \mathrm{db}$ mice $(n=5)$. b Identification of usf1 elevated in the liver of 10-week-HFD-fed mice $(n=5)$. c Expression of USF1 in the HepG2 cells treated with $300 \mu \mathrm{M}$ O/P mixture for $48 \mathrm{~h}(n=3)$. d Expression levels of USF1, uc.372, INSM2, and Nox2 in the HepG2 cells infected with Ad-USF1 or AdNC for $48 \mathrm{~h}(n=3)$. e Protein levels of ACC, FAS, SCD1, and CD36 in the HepG2 cells infected with Ad-USF1 or Ad-NC for $48 \mathrm{~h}$ (representative blots from three similar experiments) $(n=3)$. f Real-time PCR analysis of USF1, uc.372, INSM2, and Nox2 in the HepG2 cells transfected with specific siRNAs targeting USF1 or NC for $48 \mathrm{~h}(n=3)$. $\mathbf{g}$ Western blot analysis of ACC, FAS, SCD1, and CD36 expression in the HepG2 cells transfected with specific siRNAs targeting USF1 (representative blots from three similar experiments) $(n=3)$. $\mathbf{h}$ The mRNA level of uc.372 in the HepG2 cells infected with Ad-USF1 in the presence or absence of silNSM2 or NC for $48 \mathrm{~h}(n=3)$. $\mathbf{i}$ The mRNA levels of USF1 and uc.372 in the HepG2 cells infected with Ad-USF1 and Ad-uc.372i for $48 \mathrm{~h}$ ( $n$ $=3)$. $\mathbf{j}$ The mRNA levels of ACC, FAS, SCD1, and CD36 in the HepG2 cells infected with Ad-USF1 and Ad-uc.372i or Ad-NC for 48 h ( $n=3$ ). Data are mean $\pm \mathrm{SEM} ;{ }^{\star} P<0.05 ;{ }^{\star \star} P<0.01 ;{ }^{\star \star \star} P<0.001$ vs. control group. $(\mathbf{a}, \mathbf{b}, \mathbf{c}, \mathbf{d}, \mathbf{e}, \mathbf{g}$, i Student's $t$-test; $\mathbf{f}, \mathbf{h}, \mathbf{j}$ analysis of variance (ANOVA)) 
a

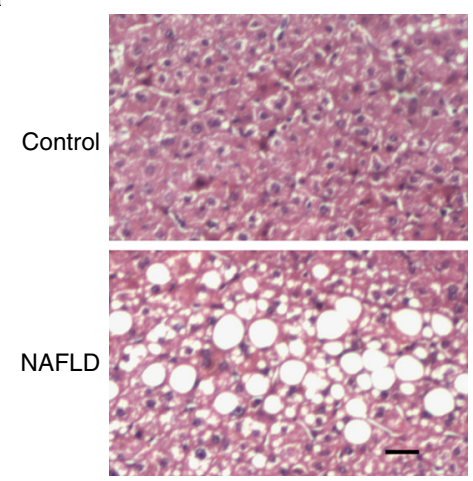

b

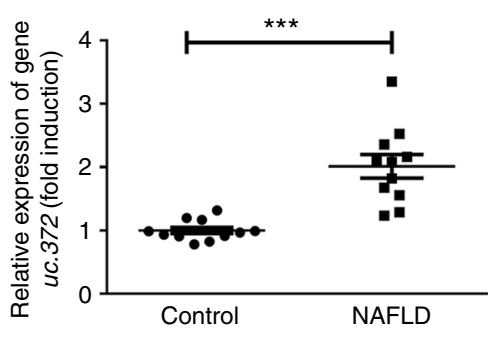

c

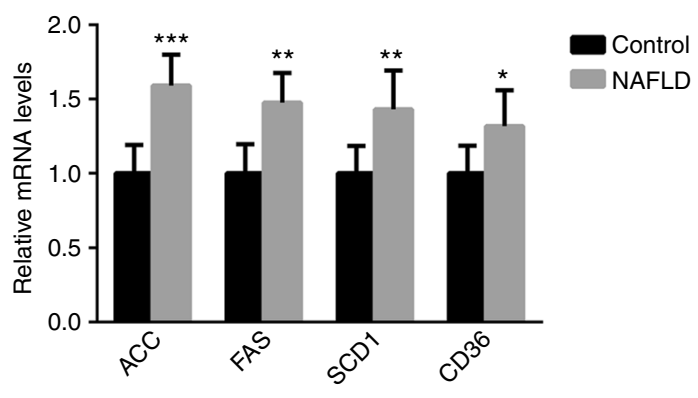

d

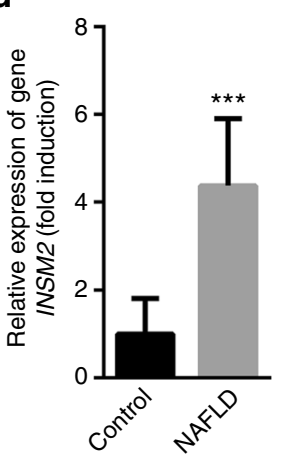

g

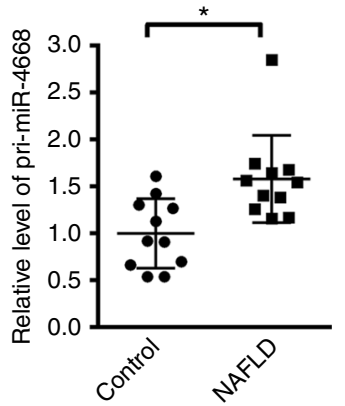

e

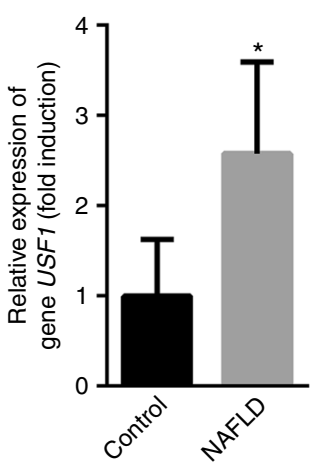

f

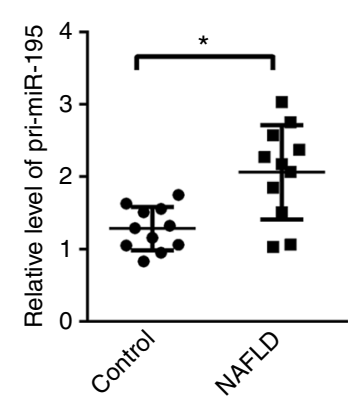

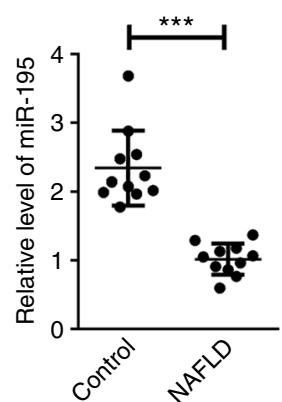

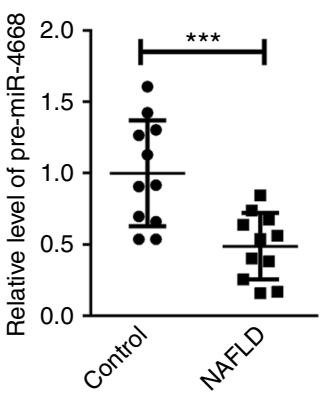

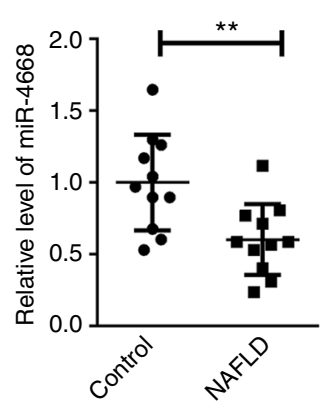

h

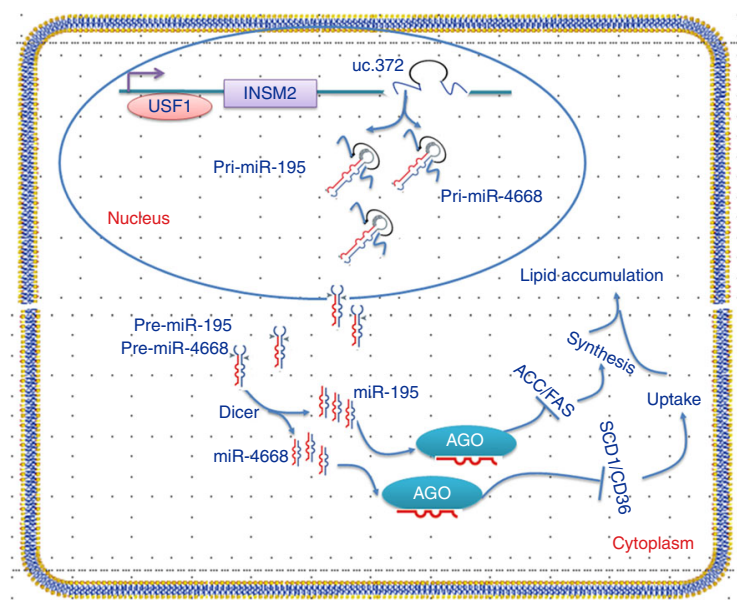

Fig. 8 Effect of uc.372 on abnormal lipid accumulation is subjected to regulation of miR-195/ miR-4668 in the liver of NAFLD patients. a Oil Red O staining of liver frozen sections from NAFLD patients or healthy control (representative image from three similar experiments). Scale bar, $100 \mu \mathrm{m}$. $\mathbf{b}$ The level of uc.372 in the liver samples of NAFLD patients or healthy control $(n=11)$. c The mRNA levels of ACC, FAS, SCD1, and CD36 in the liver samples of NAFLD patients or healthy control $(n=3)$. d The mRNA level of INSM2 in the liver samples of NAFLD patients or healthy control $(n=11)$. e The mRNA level of USF1 in the liver samples of NAFLD patients or healthy control $(n=11)$. $\mathbf{f}$ The levels of pri-miR-195, pre-miR-195, and mature miR-195 in the liver samples of NAFLD patients or healthy control $(n=11)$. $\mathbf{g}$ The levels of pri-miR-4668, pre-miR-4668, and mature miR-4668 in the liver samples of NAFLD patients or healthy control $(n=11)$. h Schematic depicting our proposed model that us.372 drives hepatic lipid accumulation. Data are mean \pm SEM; Student's $t$-test: ${ }^{\star} P<0.05 ;{ }^{\star \star} P<0.01 ;{ }^{\star \star \star} P<0.001$ vs. control group

the maturation of miR-195/miR-4668, thus relieving the suppression of target genes, including ACC, FAS, SCD1, and CD36. We also propose that uc.372 inhibitors might be potential therapeutic agents for NAFLD. In addition, we demonstrate that the expression of uc. 372 is regulated by USF1 and is dependent on the transcription of INSM2. Of note, an analysis of uc.372 distribution in various tissues identifies abundant uc.372 expression in metabolism-related organs of HFD-fed mice, including muscle, liver, heart, and fat. Therefore, a main objective for the future would be to investigate the potential functional roles of this lncRNA in different metabolism-related organs to maintain lipid homeostasis.

\section{Methods}

Experimental animals. The animals used in the experiment including 8-week-old male $\mathrm{db} / \mathrm{db}$ mice $(n=6)$ and age-matched male WT C57BL/6J mice $(n=6$; Peking University Health Science Center, Beijing, China). These mice were originally obtained from the Jackson Laboratory and fed a standard chow diet for 4 weeks. To 
establish the NAFLD model, 5-week-old male C57BL/6J mice were given free access to a standard chow diet or a HFD (D12451, 45\% kcal from fat, Research Diet, USA, http://www.researchdiets.com/opensource-diets/stock-diets) for 10 weeks in a temperature $\left(20-24^{\circ} \mathrm{C}\right)$ - and humidity-controlled $(45-55 \%)$ envir onment with a $12 \mathrm{~h} / 12 \mathrm{~h}$ light/dark cycle. Tail vein injection was carried out in HFD-fed mice with an adenoviral vector expressing uc.372 inhibitor (Ad-uc.372i) or negative control adenoviral vector (Ad-NCi). Furthermore, 6- to 8-week-old male $\mathrm{C} 57 \mathrm{BL} / 6 \mathrm{~J}$ mice fed a standard chow diet were transfected with the adenoviral vector expressing uc.372 mimic (Ad-uc.372 m) or negative control adenoviral vector (Ad-NCm) through tail vein injection. On day 7 after adenoviral vector injection, the mice were anesthetized, and blood was collected via cardiac puncture. Livers were harvested, snap-frozen in liquid nitrogen, and stored at $-80^{\circ} \mathrm{C}$ for further analysis ${ }^{59}$.

This mouse research procedures followed the guidelines of the Animal Ethics Committee at Beijing Hospital (BJMOH-201402).

Human liver specimens. Human liver biopsies from 10 patients with NAFLD and 10 healthy control subjects were performed with patient consent within the diagnostic workup of NAFLD. The application for patient-derived materials was approved by the Research Ethics Committee of Beijing You-An Hospital (BJYSE2015-35), and written consent was obtained from all patients. Clinical and biochemical characteristics of healthy individual and patients with NAFLD were analyzed ${ }^{60}$.

miRNA array analysis. Total $15 \mathrm{mg}$ RNA was isolated from the mixed samples of liver tissues from five male $\mathrm{db} / \mathrm{db}$ mice and five age-matched male WT mice or liver tissues from five NAFLD patients and five healthy controls. Total RNA was reverse-transcribed with biotin end-labeled random oligonucleotide primers. The transcsriptome for mouse lncRNAs was analyzed by the Agilent mouse lncRNA + mRNA Array V1.0 (CapitalBio; Beijing, China), while that for human lncRNAs was analyzed by Agilent human IncRNA + mRNA Array V4.0 (CapitalBio), including sense and antisense probes to all 481 human ultraconserved sequences, each spotted in duplicate. For RNA isolated from HepG2 cells, the transcriptome for human miRNA was analyzed using Agilent human miRNA Microarray V21.0 (CapitalBio)

The total RNA samples were Cy3-labeled with CapitalBio cRNA Amplification and Labeling Kit. And the miRNA samples were Cy3-labeled using the Agilent miRNA Complete Labeling and Hyb Kit, according to the manufacturer's guidelines. In strict accordance with the standard pipeline, the microarray hybridization, washing, and scanning were carried out. To analyze the array images, Agilent feature extraction software version 10.10 was applied. The GeneSpring GX software (Agilent Technologies) was used for normalization and subsequent data processing. The differentially expressed lncRNA or miRNSs were obtained using threshold values of $\geq 1.5$ - and $\leq-1.5$-fold change and a $t$-test $p$ value of 0.05 for all transcriptome comparisons.

Cell culture. Human hepatic carcinoma cell line HepG2 derived from a liver hepatocellular carcinoma of a 15 -year-old Caucasian male and murine hepatic cancer cell line Hep1-6 derived from the BW7756 mouse hepatoma that arose in a C57/L mouse were purchased from American Type Culture Collection. Both cells were cultured in Eagle's minimum essential medium (MEM; Invitrogen, Carlsbad, CA, USA) and supplemented with $10 \%$ fetal bovine serum (FBS; HyClone, Logan, UT, USA), $100 \mathrm{U} / \mathrm{ml}$ penicillin (Invitrogen), and $0.1 \mathrm{mg} / \mathrm{ml}$ streptomycin (HyClone) at $37^{\circ} \mathrm{C}$ with humidified air and $5 \% \mathrm{CO}_{2}$.

Construction of adenoviral vectors. Adenoviral vectors containing uc. 372 mimic (Ad-uc.372m), USF1 (Ad-USF1), uc.372 inhibitor (Ad-uc.372i), and control vector (Ad-NC) were constructed by Genepharma (Shanghai, China). Two shRNAs (GGAGGGATGATCCAATCTAAT and GGCAGATTGTTCTAAGTGATA) against uc.372 were selected to control for off-target effects.

Transient transfection. INSM2 siRNA, RALGAPA1 siRNA, USF1 siRNA, and their nonspecific siRNA (NC) were constructed by Genepharma. Transfection of siRNAs was performed with HiPerFect transfection reagent (Qiagen, Duesseldorf, Germany) as previously described ${ }^{61}$. Briefly, $6 \times 10^{5}$ cells were seeded in six-well plates with $2 \mathrm{ml}$ MEM culture medium containing $10 \% \mathrm{FBS}$ and antibiotics. At the same time, siRNAs or NC were mixed with HiPerFect transfection reagent and incubated at room temperature for $10 \mathrm{~min}$. Then, the complexes were transfected into HepG2 cells for $48 \mathrm{~h}$.

RNA isolation and real-time PCR. Total RNA from HepG2 cells was extracted with Trizol (Invitrogen) according to the manufacturer's instructions.

To quantify mRNA, real-time PCR was performed as previously reported ${ }^{62}$. In brief, a total of $1 \mu \mathrm{g}$ RNA was reverse-transcribed using a cDNA synthesis kit (Invitrogen) according to the manufacturer's instructions. For real-time PCR, $2 \mu \mathrm{l}$ template cDNA was mixed with $5 \mu$ l SYBR Green Supermix, $0.4 \mu$ forward primer,

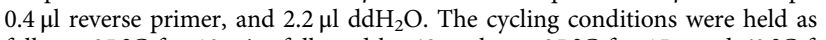
follows: $95^{\circ} \mathrm{C}$ for $10 \mathrm{~min}$, followed by 40 cycles at $95^{\circ} \mathrm{C}$ for $15 \mathrm{~s}$ and $60^{\circ} \mathrm{C}$ for min. The fluorescent signals were analyzed using an ICycler IQ5 detection system (Bio-Rad). The relative level of uc.372 was determined using the $2^{\text {-delta delta } \mathrm{Ct}}$ analysis method. $\beta$-actin was applied as the endogenous control. The primers used for real-time PCR are listed in Supplementary Table 1.

RNA-fluorescence in situ hybridization. The probes tiling the uc.372 RNA and U6 were designed following Stellaris ${ }^{\circledR}$ RNA-fluorescence in situ hybridization probe designer (Biosearch Technologies). Cell fixation, permeabilization, and hybridization to probes were performed following protocols for adherent cells. Images were acquired on a Zeiss Axio Observer Z1 Apotome fluorescence microscope.

RNA-RIP assay. RIP assay was carried out to explore the interaction between Drosha, pri-miR-195 or pri-miR-4668, and uc.372 as previously described ${ }^{63}$. Briefly, cells were seeded at a density of $1 \times 10^{5}$ cells $/ \mathrm{ml}$ for $24 \mathrm{~h}$. Then, the cells were transfected with Ad-NC or Ad-uc. $372 \mathrm{~m}$ for $48 \mathrm{~h}$. After that the cells were incubated with glycine after fixing by formaldehyde $(0.3 \%)$. The cells were washed with phosphate-buffered saline (PBS) for three times and cell suspension was reserved in RIP buffer. Then, anti-Drosha antibody (Abcam, USA) was added and incubated with the cell suspension at $37^{\circ} \mathrm{C}$ overnight. TRIzol reagent (Invitrogen) was used to isolate the precipitated RNA and the level of pri-miR-195 or pri-miR4668 was further analyzed using real-time PCR.

Luciferase reporter assay. The $3^{\prime} \mathrm{UTR}$ of SCD1 and CD36 containing the predicted binding site for miR-4668 was cloned into the pmirGLO (Promega, Madison, Wisconsin, USA) luciferase reporter vector. PCR procedures were performed as previously described. Transfection was carried out using Vigofect transfection Reagent (Vigorous Biotechnology, Beijing, China) according to the manufacturer's recommendations. Luciferase reporter assays were performed using the Dual Luciferase Reporter Assay System (Promega). Renilla activity was used as the internal control.

Northern blot. A unit of $30 \mu \mathrm{g}$ of RNA was mixed with denaturing RNA load dye (1:1, v:v, Solarbio, Beijing, China) and heated to $95^{\circ} \mathrm{C}$ for $5 \mathrm{~min}$. The RNA samples was separated on $8 \%$ denaturing Trisborate-EDTA-Urea (TBE-Urea) polyacrylamide gels and then transferred onto positively charged nylon membranes (EMD Millipore, Billerica, MA, USA). A Spectrolinker XL-1000 UV crosslinker (Xinzhi, Ningbo, China) was used for the crosslinking of RNA on the membrane at $240 \mathrm{~mJ} / \mathrm{cm}^{2}$. Then, the membranes were incubated with double-digoxigenin LNA probes in Ultrahyb Oligo hybridization buffer (Mylab Medical Technology, Beijing China) with $2 \times$ blocking reagent (Mylab Medical Technology) at $60{ }^{\circ} \mathrm{C}$ overnight. After washing with $2 \times, 0.5 \times$, and $0.1 \times$ saline sodium citrate buffer containing $0.1 \%$ SDS for $20 \mathrm{~min}$ at $60^{\circ} \mathrm{C}$, the membranes were blocked with $100 \mathrm{mM}$ maleic acid, $150 \mathrm{mM} \mathrm{NaCl}, \mathrm{pH} 7.5$, with $2 \times$ blocking reagent for $30 \mathrm{~min}$. After that the membranes were incubated with primary antibody (Mouse anti-Dig, Mylab Medical Technology) followed by three washes of PBS containing 0.1\% Tween 20 (PBS-T). The blot was further incubated with secondary antibodies (goat antimouse IGG, Mylab Medical Technology) followed by detection with chemiluminescence (Xinzhi). Probe sequences were as follows:

uc.372-5' probe, CCTTCCTATACAGCCATCCCC;

uc.372-3' probe, CTTAGAACAATCTGCCATTTGGA.

Western blot analysis. Cells or liver tissues were extracted with RIPA buffer (Solarbio). Approximately 15-30 $\mu$ g of proteins were separated by $10 \%$ SDS-PAGE and further transferred onto a polyvinylidene difluoride membrane (Millipore, Boston, MA, USA). The membrane was soaked with $8 \%$ milk in PBS-T (pH 7.5) for $2 \mathrm{~h}$ at room temperature and was incubated with the following specific primary antibodies at $4{ }^{\circ} \mathrm{C}$ overnight: anti-ACC (1:1000, \#3676, CST); anti-FAS (1:1000, \#3180, CST); anti-SCD (1:1000, \#2283, CST); anti-CD36 (1:1000, ab133625, Abcam); and anti-GAPDH (1:1000, \#5174, CST). After washing three times with PBS-T, the membrane was incubated with the horseradish peroxidase-conjugated anti-rabbit or anti-mouse secondary antibody (1:5000, Zhongshan Gold Bridge, China) for $2 \mathrm{~h}$ at room temperature. Immunodetection was conducted using the ECL Plus detection system (Millipore) according to the manufacturer's instructions. The housekeeping gene GAPDH was used as the internal control.

Triglyceride measurement. A unit of $20 \mathrm{mg}$ of liver tissues was moved to preweighed glass tube. Then, $4 \mathrm{ml} \mathrm{CHCL} / \mathrm{CH}_{3} \mathrm{OH}(2: 1)$ was added and incubated overnight at room temperature. By morning, $850 \mu \mathrm{l} 0.05 \% \mathrm{H}_{2} \mathrm{SO}_{4}$ was added and votexed. After centrifugation for $15 \mathrm{~min}$ at $2700 \mathrm{rpm}$ at room temperature, we moved supernatant and added $1 \%$ Triton $\mathrm{X}-100$. The mixture was put in vacuum

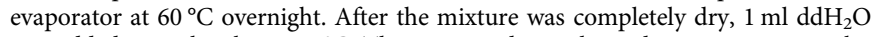
was added to each tube at $55^{\circ} \mathrm{C}$. Then, we used a triglyceride enzymatic assay kit (ShenSuoYouFu Medical Diagnostic Products Co., Ltd., Shanghai, China) to determine the contents of triglyceride.

Oil Red $\mathbf{O}$ staining. Frozen sections of liver specimens were fixed in paraformaldehyde. Then, the slides were stained with Oil Red $\mathrm{O}$ as previously described $^{64,65}$. 
Hematoxylin and eosin staining. For hematoxylin and eosin staining, the slides were first incubated with hematoxylin for $5 \mathrm{~min}$ and then washed with $1 \%$ ethanol hydrochloride for $3 \mathrm{~s}$. After washing with water, the slides were stained with eosin for $3 \mathrm{~min}$ and dehydrated with an alcohol gradient. Vacuoles were considered the lipid droplets ${ }^{65}$.

Cell fractioning. Cytoplasmic and nuclear fractions were obtained with the ProteoExtract Subcellular Proteome Extraction Kit (Calbiochem, \#539790). RNA from each fraction was extracted with TRIzol.

Statistical analysis. The data are represented as the mean \pm standard error of the mean. Comparisons between groups were analyzed by the two-tailed Student's $t$ test. For comparisons of multiple groups, one-way analysis of variance was used. Differences were considered significant at $P<0.05$.

Data availability. The microarray data discussed in this publication have been deposited in NCBI's Gene Expression Omnibus (GEO) and are accessible through GEO Series accession number GSE106366, GSE107231, and GSE107396. All data pertinent to this study are available in Supplementary Information. Additional information on these data is available from the corresponding authors.

Received: 1 December 2016 Accepted: 18 January 2018

Published online: 09 February 2018

\section{References}

1. Haga, Y. et al. Nonalcoholic fatty liver disease and hepatic cirrhosis: comparison with viral hepatitis-associated steatosis. World J. Gastroenterol. 21, 12989-12995 (2015).

2. Farrell, G. C. \& Larter, C. Z. Nonalcoholic fatty liver disease: from steatosis to cirrhosis. Hepatology 43, S99-S112 (2006).

3. Kwon, $\mathrm{H}$. et al. Inhibition of hedgehog signaling ameliorates hepatic inflammation in mice with nonalcoholic fatty liver disease (nafld). Hepatology 63, 1155-1169 (2015).

4. Goffredo, M. et al. Role of tm6sf2 rs58542926 in the pathogenesis of nonalcoholic pediatric fatty liver disease: a multiethnic study. Hepatology 63, 117-125 (2016).

5. Klair, J. S. et al. A longer duration of estrogen deficiency increases fibrosis risk among postmenopausal women with nonalcoholic fatty liver disease. Hepatology 64, 85-91 (2016).

6. Djebali, S. et al. Landscape of transcription in human cells. Nature 489 101-108 (2012).

7. Ulitsky, I. \& Bartel, D. P. Lincrnas: genomics, evolution, and mechanisms. Cell 154, 26-46 (2013).

8. Prensner, J. R. \& Chinnaiyan, A. M. The emergence of lncrnas in cancer biology. Cancer Discov. 1, 391-407 (2011).

9. Liu, M. X., Chen, X., Chen, G., Cui, Q. H. \& Yan, G. Y. A computational framework to infer human disease-associated long noncoding rnas. PLoS ONE 9, e84408 (2014)

10. Yarmishyn, A. A. \& Kurochkin, I. V. Long noncoding rnas: a potential novel class of cancer biomarkers. Front. Genet. 6, 145 (2015).

11. Lalevee, S. \& Feil, R. Long noncoding rnas in human disease: emerging mechanisms and therapeutic strategies. Epigenomics 7, 877-879 (2015).

12. Bhan, A. \& Mandal, S. S. Long noncoding rnas: emerging stars in gene regulation, epigenetics and human disease. ChemMedChem 9, 1932-1956 (2014).

13. Braconi, C. et al. Expression and functional role of a transcribed noncoding rna with an ultraconserved element in hepatocellular carcinoma. Proc. Natl Acad. Sci. USA 108, 786-791 (2011).

14. Yang, Q., Zhang, R. W., Sui, P. C., He, H. T. \& Ding, L. Dysregulation of noncoding rnas in gastric cancer. World J. Gastroenterol. 21, 10956-10981 (2015).

15. Necsulea, A. et al. The evolution of lncrna repertoires and expression patterns in tetrapods. Nature 505, 635-640 (2014).

16. Bejerano, G. et al. Ultraconserved elements in the human genome. Science 304, 1321-1325 (2004)

17. Lareau, L. F., Inada, M., Green, R. E., Wengrod, J. C. \& Brenner, S. E. Unproductive splicing of sr genes associated with highly conserved and ultraconserved DNA elements. Nature 446, 926-929 (2007).

18. McCole, R. B., Fonseka, C. Y., Koren, A. \& Wu, C. T. Abnormal dosage of ultraconserved elements is highly disfavored in healthy cells but not cancer cells. PLoS Genet. 10, e1004646 (2014).

19. Baira, E., Greshock, J., Coukos, G. \& Zhang, L. Ultraconserved elements: genomics, function and disease. RNA Biol. 5, 132-134 (2008)

20. Calin, G. A. et al. Ultraconserved regions encoding ncrnas are altered in human leukemias and carcinomas. Cancer Cell 12, 215-229 (2007).
21. Lujambio, A. et al. Cpg island hypermethylation-associated silencing of non coding rnas transcribed from ultraconserved regions in human cancer. Oncogene 29, 6390-6401 (2010).

22. Liz, J. et al. Regulation of pri-mirna processing by a long noncoding rna transcribed from an ultraconserved region. Mol. Cell 55, 138-147 (2014).

23. Mestdagh, $\mathrm{P}$. et al. An integrative genomics screen uncovers ncrna t-ucr functions in neuroblastoma tumours. Oncogene 29, 3583-3592 (2010).

24. Scaruffi, P. et al. Transcribed-ultra conserved region expression profiling from low-input total rna. BMC Genomics 11, 149 (2010).

25. Scaruffi, P. et al. Transcribed-ultra conserved region expression is associated with outcome in high-risk neuroblastoma. BMC Cancer 9, 441 (2009).

26. Singh, R., Yadav, V., Kumar, S. \& Saini, N. Microrna-195 inhibits proliferation, invasion and metastasis in breast cancer cells by targeting fasn, hmgcr, acaca and cyp27b1. Sci. Rep. 5, 17454 (2015).

27. Cai, T. et al. Expression of insulinoma-associated 2 (insm2) in pancreatic islet cells is regulated by the transcription factors ngn 3 and neurod1. Endocrinology 152, 1961-1969 (2011).

28. Iyer, M. K. et al. The landscape of long noncoding rnas in the human transcriptome. Nat. Genet. 47, 199-208 (2015).

29. Kern, A. D., Barbash, D. A., Chang Mell, J., Hupalo, D. \& Jensen, A. Highly constrained intergenic drosophila ultraconserved elements are candidate ncrnas. Genome Biol. Evol. 7, 689-698 (2015).

30. Teng, K. Y. \& Ghoshal, K. Role of noncoding rnas as biomarker and therapeutic targets for liver fibrosis. Gene Expr. 16, 155-162 (2015).

31. Sun, C. et al. Genome-wide analysis of long noncoding rna expression profiles in patients with non-alcoholic fatty liver disease. IUBMB Life 67, 847-852 (2015).

32. Li, P. et al. A liver-enriched long non-coding rna, lnclstr, regulates systemic lipid metabolism in mice. Cell Metab. 21, 455-467 (2015).

33. Hannah, W. N. \& Harrison, S. A. Nafld and elastography-incremental advances but work still to be done. Hepatology 64, 2234-2243 (2016).

34. Tilg, H., Moschen, A. R. \& Szabo, G. Interleukin-1 and inflammasomes in ald/ aah and nafld/nash. Hepatology 64, 955-965 (2016).

35. Phielix, E. et al. High oxidative capacity due to chronic exercise training attenuates lipid-induced insulin resistance. Diabetes 61, 2472-2478 (2012).

36. Samuel, V. T. \& Shulman, G. I. Mechanisms for insulin resistance: common threads and missing links. Cell 148, 852-871 (2012).

37. Cesana, M. et al. A long noncoding rna controls muscle differentiation by functioning as a competing endogenous rna. Cell 147, 358-369 (2011).

38. Dey, B. K., Pfeifer, K. \& Dutta, A. The h19 long noncoding rna gives rise to micrornas mir-675-3p and mir-675-5p to promote skeletal muscle differentiation and regeneration. Genes Dev. 28, 491-501 (2014).

39. Mueller, A. C. et al. Munc, a long noncoding rna that facilitates the function of myod in skeletal myogenesis. Mol. Cell. Biol. 35, 498-513 (2015).

40. Watts, R., Johnsen, V. L., Shearer, J. \& Hittel, D. S. Myostatin-induced inhibition of the long noncoding rna malat1 is associated with decreased myogenesis. Am. J. Physiol. Cell. Physiol. 304, C995-C1001 (2013).

41. Inokuchi-Shimizu, S. et al. Tak1-mediated autophagy and fatty acid oxidation prevent hepatosteatosis and tumorigenesis. J. Clin. Invest. 124, 3566-3578 (2014).

42. Franco-Zorrilla, J. M. et al. Target mimicry provides a new mechanism for regulation of microrna activity. Nat. Genet. 39, 1033-1037 (2007).

43. Ebert, M. S., Neilson, J. R. \& Sharp, P. A. Microrna sponges: competitive inhibitors of small rnas in mammalian cells. Nat. Methods 4, 721-726 (2007).

44. Kallen, A. N. et al. The imprinted h19 lncrna antagonizes let-7 micrornas. Mol. Cell 52, 101-112 (2013).

45. Kawahara, Y. \& Mieda-Sato, A. Tdp-43 promotes microrna biogenesis as a component of the drosha and dicer complexes. Proc. Natl Acad. Sci. USA 109, 3347-3352 (2012)

46. Choudhury, N. R. et al. Tissue-specific control of brain-enriched mir-7 biogenesis. Genes Dev. 27, 24-38 (2013).

47. Ryan, B. M., Robles, A. I. \& Harris, C. C. Genetic variation in microrna networks: the implications for cancer research. Nat. Rev. Cancer 10, 389-402 (2010).

48. Griffiths-Jones, S. The microrna registry. Nucleic Acids Res. 32, D109-D111 (2004).

49. Castilla-Llorente, V., Nicastro, G. \& Ramos, A. Terminal loop-mediated regulation of mirna biogenesis: selectivity and mechanisms. Biochem. Soc. Trans. 41, 861-865 (2013).

50. Zhang, X. \& Zeng, Y. The terminal loop region controls microrna processing by drosha and dicer. Nucleic Acids Res. 38, 7689-7697 (2010).

51. Liu, L., Chen, L., Xu, Y., Li, R. \& Du, X. Microrna-195 promotes apoptosis and suppresses tumorigenicity of human colorectal cancer cells. Biochem. Biophys. Res. Commun. 400, 236-240 (2010).

52. $\mathrm{Xu}, \mathrm{T}$. et al. Microrna-195 suppresses tumorigenicity and regulates g1/s transition of human hepatocellular carcinoma cells. Hepatology 50, 113-121 (2009). 
53. Bartel, D. P. Micrornas: target recognition and regulatory functions. Cell 136, 215-233 (2009).

54. Karginov, F. V. \& Hannon, G. J. Remodeling of ago2-mrna interactions upon cellular stress reflects mirna complementarity and correlates with altered translation rates. Genes Dev. 27, 1624-1632 (2013).

55. Hutvagner, G. \& Zamore, P. D. A microrna in a multiple-turnover rnai enzyme complex. Science 297, 2056-2060 (2002).

56. Grimson, A. et al. Microrna targeting specificity in mammals: determinants beyond seed pairing. Mol. Cell 27, 91-105 (2007)

57. Wang, D. \& Sul, H. S. Upstream stimulatory factors bind to insulin response sequence of the fatty acid synthase promoter. Usf1 is regulated. J. Biol. Chem. 270, 28716-28722 (1995).

58. Wu, S. et al. Upstream transcription factor 1 influences plasma lipid and metabolic traits in mice. Hum. Mol. Genet. 19, 597-608 (2010).

59. $\mathrm{Ng}, \mathrm{R}$. et al. Inhibition of microrna-24 expression in liver prevents hepatic lipid accumulation and hyperlipidemia. Hepatology 60, 554-564 (2014).

60. Guo, J. et al. Reduced mir-200b and mir-200c expression contributes to abnormal hepatic lipid accumulation by stimulating jun expression and activating the transcription ofsrebp1. Oncotarget 7, 36207-36219 (2016).

61. Fang, W. et al. Microrna-20a-5p contributes to hepatic glycogen synthesis through targeting p63 to regulate p53 and pten expression. J. Cell. Mol. Med. 20, 1467-1480 (2016).

62. Guo, J. et al. Mir-291b-3p induces apoptosis in liver cell line nctc1469 by reducing the level of rna-binding protein hur. Cell. Physiol. Biochem. 33, 810-822 (2014).

63. Chen, S. et al. Biological function and mechanism of malat-1 in renal cell carcinoma proliferation and apoptosis: Role of the malat-1-livin protein interaction. J. Physiol. Sci. 67, 577-585 (2016).

64. Lee, K. H. et al. Anti-obesity effects of starter fermented kimchi on 3t3-11 adipocytes. Prev. Nutr. Food Sci. 20, 298-302 (2015).

65. Kleiner, D. E. et al. Design and validation of a histological scoring system for nonalcoholic fatty liver disease. Hepatology 41, 1313-1321 (2005).

\section{Acknowledgements}

This work was supported by grants from National Basic Research Program (973 program) of China (2014CB910503 and 2012CB517502) and grants

(81700765 and 81570789) from National Natural Science Foundation of China.

\section{Author contributions}

J.G. and W.F. performed the experiments, analyzed the data, and contributed equally. L.S., Y.L., and L.D. collected the biopsy samples and performed the bioinformatics analyses. X.H. and W.T. performed the experiments. L.Y., and J.L. conceived and supervised the study and wrote the manuscript.

\section{Additional information}

Supplementary Information accompanies this paper at https://doi.org/10.1038/s41467018-03072-8.

Competing interests: The authors declare no competing financial interests.

Reprints and permission information is available online at http://npg.nature.com/ reprintsandpermissions/

Publisher's note: Springer Nature remains neutral with regard to jurisdictional claims in published maps and institutional affiliations.

\section{(c) (1)}

Open Access This article is licensed under a Creative Commons Attribution 4.0 International License, which permits use, sharing, adaptation, distribution and reproduction in any medium or format, as long as you give appropriate credit to the original author(s) and the source, provide a link to the Creative Commons license, and indicate if changes were made. The images or other third party material in this article are included in the article's Creative Commons license, unless indicated otherwise in a credit line to the material. If material is not included in the article's Creative Commons license and your intended use is not permitted by statutory regulation or exceeds the permitted use, you will need to obtain permission directly from the copyright holder. To view a copy of this license, visit http://creativecommons.org/ licenses/by/4.0/.

(c) The Author(s) 2018 